\title{
Model-Free Deconvolution of Femtosecond Kinetic Data
}

\author{
Ákos Bányász ${ }^{\dagger}$ and Erno Keszei* \\ Eötvös University Budapest, Department of Physical Chemistry, 1518 Budapest 112, P.O. Box 32, Hungary
}

Received: December 23, 2005; In Final Form: February 21, 2006

\begin{abstract}
Though shorter laser pulses can also be produced, pulses of the $100 \mathrm{fs}$ range are typically used in femtosecond kinetic measurements, which are comparable to characteristic times of the studied processes, making detection of the kinetic response functions inevitably distorted by convolution with the pulses applied. A description of this convolution in terms of experiments and measurable signals is given, followed by a detailed discussion of a large number of available methods to solve the convolution equation to get the undistorted kinetic signal, without any presupposed kinetic or photophysical model of the underlying processes. A thorough numerical test of several deconvolution methods is described, and two iterative time-domain methods (Bayesian and Jansson deconvolution) along with two inverse filtering frequency-domain methods (adaptive Wiener filtering and regularization) are suggested to use for the deconvolution of experimental femtosecond kinetic data sets. Adaptation of these methods to typical kinetic curve shapes is described in detail. We find that the modelfree deconvolution gives satisfactory results compared to the classical "reconvolution" method where the knowledge of the kinetic and photophysical mechanism is necessary to perform the deconvolution. In addition, a model-free deconvolution followed by a statistical inference of the parameters of a model function gives less biased results for the relevant parameters of the model than simple reconvolution. We have also analyzed real-life experimental data and found that the model-free deconvolution methods can be successfully used to get undistorted kinetic curves in that case as well. A graphical computer program to perform deconvolution via inverse filtering and additional noise filters is also provided as Supporting Information. Though deconvolution methods described here were optimized for femtosecond kinetic measurements, they can be used for any kind of convolved data where measured experimental shapes are similar.
\end{abstract}

\section{Introduction}

Deconvolution in chemistry dates back to the early 1930s. The first (linear iterative) deconvolution method was originally described in a physics journal ${ }^{1}$ and used mostly to "sharpen" convolved experimental spectral lines. With the advent of reasonably fast computers and the fast Fourier transform (FFT), this had been replaced by the linear deconvolution method based on Fourier transforms, 2,3 which is usually called "inverse filtering". ${ }^{4}$ The need for deconvolution also emerged in the evaluation of pulse radiolysis, flash photolysis, and later laser photolysis results, when studied kinetic processes were so fast that reaction times were comparable to the temporal width of the pulse or lamp signals. ${ }^{5}$ A number of methods have been used ever since to get the deconvolved kinetic signal. A critical review of the deconvolution methods is described in two papers, ${ }^{6,7}$ which deal mostly with the evaluation of nanosecond to picosecond time scale fluorescence or luminescence data, collected usually by single photon counting techniques.

The availability of femtosecond pulse lasers led to the development of femtochemistry, ${ }^{8}$ where the time window enables the very detection of the transition state in an elementary reaction. However, due to the very small time window of the measurement, a typical femtosecond kinetic trace usually contains fewer data points than picosecond kinetic traces. All deconvolution methods are very much sensitive to experimental noise, so the "classical" deconvolution methods described in

* Corresponding author. E-mail address: keszei@chem.elte.hu

$\dagger$ Also at the Research Institute for Solid State Physics and Optics of the Hungarian Academy of Sciences, P.O. Box 49, H 1525 Budapest, Hungary. refs 1-7 usually fail when applied to femtosecond kinetic data. We have been working on developing model-free deconvolution methods that can be used to deconvolve ultrafast kinetic traces. Our preliminary results using inverse filtering have been published in two short papers. ${ }^{9,10}$

In this paper we describe the application of nonlinear deconvolution methods that we successfully use for model-free deconvolution of femtosecond kinetic traces, based on a large number of further computer experiments and data analysis since the publication of refs 9 and 10. In the next section, we explain the details of the procedure of ultrafast laser kinetic measurements leading to the detected convolved kinetic traces. In the following section we outline the mathematical background of nonparametric deconvolution methods that might be used to evaluate femtosecond kinetic data, followed by their implementation and numerical testing on synthetic (simulated) kinetic traces. Finally, we show deconvolution results of measured femtosecond kinetic data.

\section{Convolution of the Detected Femtosecond Pump-Probe Signals}

Elementary chemical reactions occur on the time scale of molecular vibrations, so they can only be followed with subpicosecond time resolution. Electronic devices with their top frequency of about $10 \mathrm{GHz}$ cannot follow changes at this time scale. The necessary resolution is easily achieved using the femtosecond pump-probe techniques, ${ }^{8,11}$ where reaction times are coded in delay time between a pump pulse that initiates the reaction and a probe pulse that probes the spectroscopic 


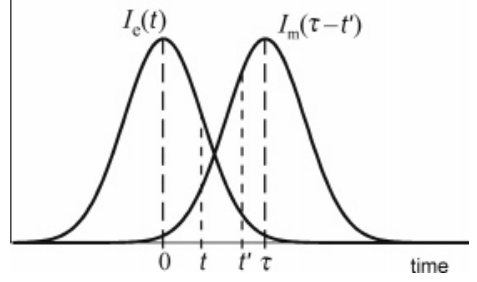

Figure 1. Notation used to describe the convolution integral detected in femtosecond pump-probe kinetic measurements. $I_{\mathrm{e}}(t)$ is the pump ("exciting") pulse and $I_{\mathrm{m}}\left(\tau-t^{\prime}\right)$ is the probe ("measuring") pulse intensity profile.

properties of the reactive system, while an integrated signal of the detector is measured with the usual speed of the relevant electronic device. Delay times on the femtosecond scale are readily obtained by varying the optical path length of either the pump or the probe beam. Considering the velocity of light in air, a difference of $0.3 \mu \mathrm{m}$ in path length results in approximately $1 \mathrm{fs}$ in time delay. As both the pump and the probe pulses should have a limited energy range (spectral width) to get reasonable selectivity both in excitation and in detection, the temporal width of the pulses cannot be too small, due to the limitation imposed by the uncertainty relation. ${ }^{12}$ The usual spectral width of about $5 \mathrm{~nm}$ in the visible range corresponds to about 100 fs transform limited (minimal) pulse width. If characteristic times of the studied reactions are in the few hundreds of femtoseconds time scale, the convolution of the "instantaneous" kinetic response with the pump and the probe pulse profiles can severely distort the detected signal.

The description of this convolution in quantum mechanical terms is extensively discussed in a recent paper. ${ }^{13}$ Here we show the convolution in terms of the experiments and measurable signals. For the sake of simplicity, let us deal with absorption measurements. A transient absorption can be described by Beer's law:

$$
I=I_{0} \times 10^{-\epsilon c 1}=I_{0} \mathrm{e}^{-\ln 10 \epsilon c l}
$$

where $I_{0}$ is the detected light intensity without excitation and $I$ is the detected light intensity after excitation, $\epsilon$ is the (decadic) molar absorptivity coefficient, $c$ is the concentration of the transient species generated by the excitation, and $l$ is the optical path length in the absorbing medium. If $\epsilon \mathrm{cl}$ is small, then the exponential can be replaced by its Taylor series up to the firstorder term:

$$
I \approx I_{0}(1-\ln 10 \epsilon c l)
$$

To make use of this approximation, differential optical densities (denoted by $\Delta \mathrm{OD}$ ) are usually detected:

$$
\Delta \mathrm{OD}=\frac{I_{0}-I}{I_{0}}=1-10^{-\epsilon c l}
$$

which can be written using the approximation (2) as

$$
\Delta \mathrm{OD} \approx 1-(1-\ln 10 \epsilon c l)=\ln 10 \epsilon c l
$$

If $\Delta \mathrm{OD} \approx 0.1$, the difference between the exact expression (3) and the approximation (4) is below $0.5 \%$, so we can use the linear approximation. To apply this result in the detection of femtosecond kinetic traces, let us consider two pulses as shown in Figure 1.

When exciting with the pulse $I_{\mathrm{e}}(t)$ and measuring the temporal evolution of absorption with the pulse $I_{\mathrm{m}}^{0}\left(\tau-t^{\prime}\right)$, which arrives into the reaction mixture after a delay of $\tau$, then we can detect at $t^{\prime}$ for the reaction initiated at time $t$ the instantaneous absorption response

$$
I_{\mathrm{inst}}\left(\tau, t, t^{\prime}\right)=I_{\mathrm{m}}^{0}\left(\tau-t^{\prime}\right) \times 10^{-\epsilon c\left(t, t^{\prime}\right) l}
$$

or, substituting the linear approximation into the expression for $\triangle O D$ in eqs (3) and (4),

$$
\begin{aligned}
& \Delta \mathrm{OD}_{\mathrm{inst}}\left(\tau, t, t^{\prime}\right)= \\
& \quad \frac{I_{\mathrm{m}}^{0}\left(\tau-t^{\prime}\right)-I_{\mathrm{m}}^{0}\left(\tau-t^{\prime}\right)\left(1-\ln 10 \epsilon c\left(t, t^{\prime}\right) l\right)}{I_{\mathrm{m}}^{0}\left(\tau-t^{\prime}\right)}=\ln 10 \epsilon c\left(t, t^{\prime}\right) l
\end{aligned}
$$

However, as $I_{\mathrm{m}}^{0}\left(\tau-t^{\prime}\right)$ is time dependent, the quantity $\ln 10 \epsilon c l$ is detected with time-dependent "weights". Let us introduce $I_{\mathrm{m}}\left(\tau-t^{\prime}\right)$ as $I_{\mathrm{m}}^{0}\left(\tau-t^{\prime}\right)$ divided by its integral between $-\infty$ and $+\infty$. Obviously, the function $I_{\mathrm{m}}\left(\tau-t^{\prime}\right)$ has the following property:

$$
\int_{-\infty}^{\infty} I_{\mathrm{m}}\left(\tau-t^{\prime}\right) \mathrm{d} t^{\prime}=1
$$

and can be considered as the detection probability density function at time $t^{\prime}$. Using this function, we can write the integral of $\Delta \mathrm{OD}$ detected for the excitation at time $t$ as

$$
\Delta \mathrm{OD}(\tau, t)=\ln 10 \int_{-\infty}^{\infty} I_{\mathrm{m}}\left(\tau-t^{\prime}\right) \epsilon c\left(t^{\prime}-t\right) l \mathrm{~d} t^{\prime}
$$

We emphasize again that $I_{\mathrm{m}}\left(\tau-t^{\prime}\right)$ is no more the detected intensity of the probe pulse prior to excitation but the detection probability density. (As the intensity cancels in eq (6), its absolute value is not needed in further calculations.) Similarly, if $I_{\mathrm{e}}(t)$ denotes the probability density function derived from the absolute intensity of the pump pulse normalized to unit integral, the total concentration present at time $t^{\prime}$ generated by the entire exciting (pump) pulse can be written as

$$
c\left(t^{\prime}\right)=\int_{-\infty}^{t^{\prime}} I_{\mathrm{e}}(t) c\left(t^{\prime}-t\right) \mathrm{d} t
$$

Redefining the function $c\left(t^{\prime}-t\right)$ so that $c\left(t^{\prime}-t\right)=0$ for all $t>t^{\prime}$ (this is the usual kinetic definition; the concentration change is zero for negative reaction times), we can write eq (9) in a form that is more common in probability theory:

$$
c\left(t^{\prime}\right)=\int_{-\infty}^{\infty} I_{\mathrm{e}}(t) c\left(t^{\prime}-t\right) \mathrm{d} t
$$

This expression can more easily be treated in mathematical terms as well, as the integral obtained combining eqs (8) and (10),

$$
\Delta \mathrm{OD}(\tau)=\ln 10 \int_{-\infty}^{\infty} I_{\mathrm{m}}\left(\tau-t^{\prime}\right) \int_{-\infty}^{\infty} I_{\mathrm{e}}(t) \epsilon c\left(t^{\prime}-t\right) l \mathrm{~d} t \mathrm{~d} t^{\prime}
$$

is easy to be written as a convolution. The convolution of the functions $f$ and $g$ is defined ${ }^{14}$ as

$$
f \otimes g=\int_{-\infty}^{\infty} f(t) g(\tau-t) \mathrm{d} t
$$

The correlation of the functions $g$ and $h$ can be written ${ }^{14}$ as

$$
\operatorname{corr}(g, h)=\int_{-\infty}^{\infty} g(\tau+t) h(t) \mathrm{d} t
$$


This latter can also be written as a convolution integral if we use $\tilde{g}(\tau-t)$, the function $g(\tau+t)$ with its inverted time scale:

$$
\operatorname{corr}(g, h)=\int_{-\infty}^{\infty} g(\tau+t) h(t) \mathrm{d} t=\int_{-\infty}^{\infty} \tilde{g}(\tau-t) h(t) \mathrm{d} t=
$$

If there are more than one transient species absorbing at the probe wavelength, we should sum the contributions of all the $n$ absorbing species writing $\sum_{k=1}^{n} \epsilon_{k} c_{k} l$ in place of $\epsilon c l$. Making use of the fact that the order of integration is immaterial (or of the commutability and associability of convolution) we can write

$$
\Delta \mathrm{OD}(\tau)=\left[\operatorname{corr}\left(I_{\mathrm{m}}, I_{\mathrm{e}}\right)\right] \otimes\left(\ln 10 \sum_{k=1}^{n} \epsilon_{k} c_{k} l\right)
$$

The first function on the right side can be considered as an "effective pulse" which in practice also includes a time broadening due to the difference of the refractive indices at the pump and the probe wavelengths, also called group velocity mismatch.

Summarizing the above results we can state that the detected integrated differential optical density is the convolution of the effective pulse with the instantaneous transient differential optical density $\ln 10 \sum_{k=1}^{n} \epsilon_{k} c_{k} l .{ }^{15}$ This result is obtained for transient absorption measurements. However, if fluorescence emission is measured, the intensity of the detected signal is proportional to the probe or gate beam intensity $I_{\mathrm{m}}$, which excites the fluorescing species or creates the sum frequency. Consequently, the same formalism applies, except for a proportionality constant.

Let us reformulate eq (15) in terms of the usual notation of imaging. Thus, $\Delta \mathrm{OD}(\tau)$ is called the image function $i(t)$, the effective pulse is the spread function $s(t)$, and the instantaneous response function is the object function $o(t)$. It is this latter we want to infer from the measured data. The above convolution can then be rewritten as

$$
i=s \otimes o
$$

\section{Deconvolution Methods}

The information on the kinetic and photophysical behavior in a femtochemical experiment is contained in the undistorted function $o(t)$. To get this function from the detected (convolved) signal $i(t)$, the spread function $s(t)$ should be known and the integral eq (16) should be solved. This procedure yielding the original $o(t)$ function is called deconvolution. Equation (16) can also be used to determine the effective pulse $s(t)$. This procedure is called identification, but it is not the subject of this paper. Moreover, as there are methods that enable direct experimental detection of $s(t)$ even for multiphoton excitation experiments, ${ }^{16}$ identification is not of great importance in femtochemistry.

There exist different groups of deconvolution methods used in treating laser photolysis data. The first widely used group can be called reconvolution. Least-squares iterative reconvolution is the most widely used version. ${ }^{6,11}$ Strictly speaking, this is not a deconvolution, but a least-squares fitting of a suitable model function convolved with the effective pulse to the measured (convolved) $i(t)$ data, thus estimating kinetic and photophysical parameters. Once the parameters are known, the (nonconvolved) $o(t)$ function can also be reconstructed. Somewhat similar is the method of moments (another parameter estimation method), which only works for multiexponential decay models, being inferior to the least-squares method that can treat any kinetic model. Other iterative methods based on either Laplace or Fourier transforms work also only for multiexponential decay models. The method of modulating functions is an elegant, noniterative method, which provides the parameters of multiexponential decay by solving integral equations. There are only two methods leading primarily to a deconvolved dataset $o(t)$ which have been used in laser photolysis. One is the so-called exponential series method. It is based on the assumption that laser photolysis measurements can be interpreted as the sum of a few exponentials. This method does not suppose that the sum of exponentials would have any physical meaning. Accordingly, time constants are fixed when using this method, and a least-squares estimation of the amplitudes is performed so that the convolution of the exponential series with the effective pulse matches the measured (convolved) $i(t)$ function. The nonconvolved series then represents the nonconvolved $o(t)$ function. The other direct deconvolution method is based on inverse filtering using Fourier transforms, with special noise handling derived from the Poissonian error of the data and an exponential extrapolation of the dataset. All the aforementioned methods and the relevant original papers are described in the references. ${ }^{6,7}$

We can summarize that none of the above methods are completely free of (usually arbitrary) models, most of them requiring a multiexponential decay of the species formed by excitation with the pump pulse. However, femtochemical processes, especially in condensed media, are usually more complicated and cannot be described as simple sums of exponential functions. Iterative least squares is always a reasonable choice, if we have a priori knowledge of a reaction mechanism and related photodynamic properties. Unfortunately, in most of the cases, we do not have it. Therefore, it would be useful to be able to directly deconvolve the measured $i(t)$ function. The deconvolved $o(t)$ may be more instructive for the chemist to find out possible mechanisms, and testing the mechanisms would need much less computation without the convolution involved in the model function. In addition, if a reliable deconvolution can be obtained, the additional uncertainty of the model parameters due to the correlation with the effective pulse parameters, always present in an iterative reconvolution procedure, could be avoided. (Though the functional form of the effective pulse can be measured, its exact width and "zero time" should usually be fine-tuned when evaluating ultrafast kinetic data.)

The aim of this paper is to discuss direct, model-free (and hence nonparametric) deconvolution methods, so we do not deal further with those mentioned above. In a direct deconvolution, the solution of the convolution eq (16) provides a reconstructed $\hat{o}(t)$ dataset from the measured $i(t)$ data so that

$$
i=s \otimes \hat{o}
$$

Many direct deconvolution methods are described in the comprehensive monograph edited by Jansson. ${ }^{17}$ Other useful reviews are the invited paper of Schafer et al., ${ }^{18}$ or a more recent tutorial paper on several advanced methods in the field of image reconstruction. ${ }^{19}$

Before describing particular methods that we have used successfully to deconvolve femtochemical data, we would like to point out some major difficulties in the reconstruction of the undistorted function $o(t)$. One of the problems is that of the uniqueness of solution of the integral eq (16). Suppose that the solution $\hat{o}(t)$ is not unique in the sense that we can find other solutions of the form

$$
\hat{o}(t)=o(t)+w(t)
$$


where $w(t)$ is the part of $\hat{o}(t)$ which makes the original $o(t)$ become a spurious function. If there exist some functions $w(t)$ that fulfill the condition

$$
\int_{-\infty}^{\infty} s(\tau-t) w(t) \mathrm{d} t=0
$$

or, in other words, whose convolution with the spread function $s(t)$ is zero, then $w(t)$ can be summed with the real object function $o(t)$ to give a valid solution $\hat{o}(t)$. As we shall see later, spurious components in the solution $\hat{o}(t)$ often appear.

Another problem is encountered due to experimental errors in the form of noise which adds to the "true" value of the measured $i(t)$ function, so that

$$
i(t)=i_{\text {true }}(t)+n(t)
$$

The problem of noise is also a severe limitation on the solution of the convolution equation. For details see the discussion of eqs (26) and (27).

(a) Linear Deconvolution Methods. A deconvolution method is called linear if it includes only linear operations on the input data $i(t)$ and $s(t)$. Though linear methods are not really effective to deconvolve femtosecond kinetic data, it is worth treating them as they offer an easy formal description of the deconvolution problem. In practice, kinetic measurements provide sampled quantized data of the $i(t)$ function, i.e., a finite set of data truncated numerically to some decimal digits. To describe these data, we can rewrite the convolution eq (16) in discrete form:

$$
i_{n}=\sum_{m} s_{n-m} o_{m}
$$

where the limits of $m$ comprise the entire range where $s$ has a nonzero value. The values of $n$ are those for which measured data are available. We can rewrite this equation in matrix formulation:

$$
\mathbf{i}=\mathbf{S o}
$$

where $\mathbf{i}$ and $\mathbf{o}$ are column vectors of $N$ elements, and $\mathbf{S}$ is an $N \times N$ square matrix. In practice, $N$ is usually the length of the $i_{n}$ dataset, as the length of nonzero elements of the $s_{n-m}$ data is less than $N$. The elements of the $\mathbf{S}$ matrix are $s_{n, m}=s_{n-m}$ within the range of the spread function, and zero outside. From the time-independent shift property of the $s$ function we can find out that the $\mathbf{S}$ matrix should have a special property; each row is the same as the row above, except that it is (circularly) shifted one element to the right. Such a matrix is called a Toeplitz matrix.

Obviously, eq (22) represents a set of linear equations with the unknowns $o_{m}$, and we should be able to obtain the solution by simply inverting the matrix $\mathbf{S}$. However, the special structure of the Toeplitz matrix makes the solution extremely sensitive even to slight numerical imprecision, as its rows are almost identical. When calculating the inverse, very small differences of large numbers should be handled. Even if the inverse can be calculated with robust iterative methods, the solution $\hat{o}$ obtained contains large spurious fluctuations as a consequence of the experimental noise in the $\mathbf{i}$ vector.

Iterative solutions of eq (22) are promising to suppress the spurious fluctuations and noise amplification, as they could be controlled during the iterative process. Iteration is a recurrent approximation of the solution that can be written as

$$
\hat{o}_{n}^{(k+1)}=\hat{o}_{n}^{(k)}+\lambda\left(i_{n}-\sum_{m} s_{n-m} \hat{o}_{m}^{(k)}\right)
$$

where $\hat{o}^{(k+1)}$ is the next approximation with respect to $\hat{o}^{(k)}$ and $\lambda$ is called the relaxation factor. In a linear iteration method, $\lambda$ should be independent of $i, s, \hat{o}^{(k)}$, and $t$. In the "classical" van Cittert method, $\lambda=1$, and the first approximation $\hat{o}^{(0)}=i .{ }^{1} \mathrm{As}$ the iteration proceeds, the correction term becomes smaller, and convergency to the true object $o$ can be achieved. However, van Cittert's method also amplifies noise with each iteration step and leads to ever increasing spurious fluctuations in the solution $\hat{o}$, similarly to other linear methods, thus never leading to convergency. We shall show nonlinear iterations in the next section that efficiently treat this problem.

There is another obvious possibility to treat deconvolution, namely, digital filtering. A nonrecursive digital filter is defined by a convolution similar to eq (21). ${ }^{4}$ Accordingly, we can say that the measured function $i$ is obtained by digitally filtering the object $o$ with the spread function $s$. The procedure of getting back the object is called inverse filtering, which is more convenient to treat in the frequency domain, using the Fourier transforms $F(v)$ of the respective time domain functions $f(t)$.

According to the convolution theorem, ${ }^{3,4}$ eq (16) that describes the distortion can be written in the frequency domain as

$$
I(v)=S(v) O(v)
$$

where $v$ is the frequency (per unit time). ${ }^{3}$ Obviously, as convolution (i.e., digital filtering) becomes simple multiplication in the frequency domain, we can readily get the inverse filtered result

$$
O(v)=\frac{1}{S(v)} I(v)
$$

where $1 / S(v)$ is the searched-for inverse filter. The procedure of inverse filtering to get the object $o$ is in principle straightforward. We should Fourier transform both the measured image function $i$ and the spread function $s$, divide the transform of the first with that of the second, and inverse Fourier transform the result $O(v)$ to get $o(t)$ : in principle, but not in practice. As the reader might have noticed, inverse filtering as described here is also a linear transformation of the measured $i$ function, so we can expect the usual spurious fluctuations and noise amplification in the solution $\hat{o}$. As we shall see later, these fluctuations become many orders of magnitude larger than the function $o$ itself. This is due to the fact that the slowly varying spread function has nonzero components only close to $v=0$, so applying the inverse filtering according to eq (25) means dividing at higher frequencies virtually by zero, largely amplifying the high-frequency noise.

However, digital filtering in the frequency domain makes it easy both to describe and to treat the effect of noise. We can rewrite eq (20) as

$$
I(v)=I_{\text {true }}(v)+N(v)
$$

Inverse filtering of $I(v)$ then becomes

$$
\hat{O}(v)=\frac{I_{\text {true }}(v)}{S(v)}+\frac{N(v)}{S(v)}
$$

As the measured optical density in kinetics is usually a slowly varying function (even more so after convolution with the effective pulse), it has nonzero values mostly close to zero frequency. The noise content of the measured data typically has high-frequency components as well, which means that the highfrequency part of the solution $\hat{O}(v)$ is dominated by the noise. 
Division of the nonzero $N(v)$ values at higher frequencies by $S(v)$-where this latter has virtually zero values, as mentioned before-further enhances the noise content of $\hat{O}(v)$. This is the origin of the discouraging result of simple inverse filtering, that the amplitude of noise in the inverse Fourier transform $\hat{o}(t)$ largely exceeds the amplitude of the signal itself.

Analysis of eq (27) also offers the clue to get rid of the amplified noise. All we have to do, at least in principle, is to "cut" the high frequencies of $\hat{O}(v)$ before inverse transformation so that we get rid of the amplified noise, but keep the lowfrequency part that contains the information necessary to reconstruct $\hat{o}(t)$ the most closely resembling the true object. If such a low-pass filter is a linear filter only, the overall procedure is still a linear transformation. The success of getting rid of the noise this way depends on the extent of overlapping of the $I_{\text {true }}(v)$ and the $N(v)$ functions. If nonzero amplitudes of the two functions occur in distinct frequency regions, noise filtering can be very efficient. If the two regions overlap, there is a trade-off between noise reduction and object distortion. In this case, additional noise filtering can be used simultaneously with inverse filtering. Filter parameters are then optimized to get the optimal deconvolution result.

Effective noise filtering can also be done in the time domain in some form of "smoothing" the experimental $i_{n}$ dataset prior to deconvolution. This can be done with a wide variety of filters, ${ }^{3,4,17}$ but they should be applied with caution, as they typically distort the true object function as well. A safe method that works well without distortion is the so-called reblurring procedure proposed by Kawata et. al. ${ }^{20,21}$ In this procedure, both spread and image are convolved with the inverted time-scale spread function $s(-t)$. Following this, the starting image will be the correlation $s(-t) \otimes i(t)$, and the spread function used in the deconvolution will be the autocorrelation $s(-t) \otimes s(t)$. The noise in the measured $i(t)$ is largely reduced by this smoothing, resulting also in an effective damping of the spurious fluctuations in the solution $\hat{o}(t)$, but the number of iterations necessary to obtain convergency is much higher after reblurring. To overcome this problem, a variable convergence speed method has also been proposed. ${ }^{22}$ However, the increased computational requirement does not lead to inconvenience nowadays, due to recently available high-speed processors.

(b) Constrained Nonlinear Deconvolution Methods. Appearance of spurious components and noise amplification can easily result in complete failure of the reconstruction of the object function, or in its heavy distortion. We can greatly improve the result of reconstruction if we make use of our prior knowledge of the object function. If we know the functional form except for a few parameters, a least-squares estimate of those parameters with the help of the convolved function usually results in a completely smooth function. Even if we do not know the kinetic model function, when measuring physical signals, we usually know some useful properties of the measured functions a priori, which properties can easily be implemented in iterative procedures. To include constraints and nonlinearity in the iteration eq (23), we can write ${ }^{17,18}$

$$
\hat{o}^{(k+1)}=C \hat{o}^{(k)}+\lambda\left(i-s \otimes C \hat{o}^{(k)}\right)
$$

where $C$ is a constraint operator, and $\lambda$ may be dependent on $i$, $\hat{o}$, or $t$. In femtosecond kinetic applications, we can use several constraints. As already mentioned in connection with eqs (9) and (10), a transient $\triangle O D$ should be zero prior to excitation. If nonzero elements of the object function are confined within $t_{1}$ and $t_{2}$, it is called a finite extent $t^{17}$ or finite support ${ }^{18}$ constraint.
In the case of a transient $\Delta \mathrm{OD}$, we may use a semifinite support constraint, which means that we always set $\hat{o}^{(k)}(t)$ values to zero if $t<t_{1}$, the onset time of excitation in the instantaneous kinetic response. However, if we do not know the exact zero delay between the pump and the probe pulses, application of this constraint might not be useful. The positivity constraint is also applicable if there is no bleaching present in the measured $\triangle \mathrm{OD}$, or if we measure a transient fluorescence signal. This constraint forces the amplitude of the $\hat{o}(t)$ function to remain always nonnegative.

Constraints on amplitude may be implemented also in the relaxation factor $\lambda$. An elegant and rather effective formulation is the relaxation proposed by Jansson. ${ }^{23}$ His version of the nonlinear iterative method in a discrete implementation can be written as

$$
\hat{o}_{n}^{(k+1)}=\hat{o}_{n}^{(k)}+r\left(\hat{o}^{(k)}\right)\left[i_{n}-\sum_{m} s_{n-m} \hat{o}_{m}^{(k)}\right]
$$

The relaxation function $r\left(\hat{o}^{(k)}\right)$ is defined to respect the (a priori known) boundaries of the object function. If $\hat{o}_{\min }$ is the minimum and $\hat{o}_{\max }$ is the maximum of the physically acceptable values, then a general expression for the relaxation function can be given as

$$
r\left(o^{(k)}\right)=r_{0}-\frac{2 r_{0}}{\hat{o}_{\text {max }}-\hat{o}_{\min }}\left|\hat{o}^{(k)}-\frac{\hat{o}_{\text {min }}+\hat{o}_{\text {max }}}{2}\right|
$$

(Note that the original formula of Jansson ${ }^{17}$ is different; it is valid only if $\hat{o}_{\min }$ equals zero and $\hat{o}_{\max }$ is positive, while eq (30) does not require these conditions.) This function is trigonally shaped, with its maximum $r_{0}$ in the middle of the interval $\left(\hat{o}_{\mathrm{min}}\right.$; $\left.\hat{o}_{\max }\right)$, decreasing linearly with the distance from the maximum. It has zero values (no correction) at the boundaries, and becomes negative outside the physically acceptable interval. Its effect during the iterative procedure is to apply a negative correction if the amplification of noise resulted in physically nonacceptable data. Physical boundaries are often easy to fix, especially in spectroscopy. Transmittance data, for example, should lie between 0 and 1 . The method is clearly superior to simple "clipping" at the boundaries. This can easily be shown at the zero limit. If we simply clip negative values to zero, according to eq (28), they should remain zero during further iteration, while the more flexible Jansson algorithm allows subsequent relaxation from zero values.

Another group of iterative methods uses multiplicative corrections instead of additive ones. The method has been first proposed by Gold. ${ }^{24}$ The iteration equation can be written as

$$
\hat{o}_{n}^{(k+1)}=\hat{o}_{n}^{(k)} \frac{i_{n}}{\sum_{m} s_{n-m} \hat{o}_{m}^{(k)}}
$$

If both $s$ and $i$ are positive, $\hat{o}^{(k)}$ cannot become negative during this iteration. Obviously, the multiplicative correction term $i /\left(s \otimes \hat{o}^{(k)}\right)$ goes to unity if convergency is obtained. It is interesting to note that Gold's ratio method can be considered as Jansson's method using the special relaxation function $r\left[\hat{o}^{(k)}\right]=\hat{o}^{(k)} /\left(s \otimes \hat{o}^{(k)}\right) .{ }^{25}$ Note that, in addition to the dependence on the value of $\hat{o}^{(k)}$ in the Jansson method, this relaxation function also depends on the iteration number $k$. Another interesting and efficient multiplicative correction has been developed inspired by the Bayesian method of probability 
calculation, ${ }^{26}$ hence it is usually called Bayesian deconvolution. ${ }^{27}$ (Note that some authors also call it as Richardson-Lucy method.) This iteration procedure can be written as

$$
\hat{o}_{n}^{(k+1)}=\hat{o}_{n}^{(k)} \sum_{m} s_{n+m}\left(\frac{i_{n}}{\sum_{m} s_{n-m} \hat{o}_{m}^{(k)}}\right)
$$

In fact, this is an enhanced noise suppression method, where the simple Gold's correction term is filtered with the reverse time-scale spread function $s(-t)$, though the number of iterations increases substantially. This iteration also guarantees the conservation of positive values. There is also a direct use of the Bayesian estimation method in deconvolution if the object function can be interpreted as a probability distribution, which is typically not the case in femtochemistry.

A quantitative comparison of time domain iterative deconvolution methods used in spectrometry and chromatography has found Jansson's method the best performing one. ${ }^{28}$ There is an interesting study on the application of nonparametric deconvolution methods to differential calorimetric results at a scale of a few seconds. ${ }^{29}$ This kinetically motivated work concludes that constrained iterative methods with subsequent noise filtering give the most suitable results.

As we have already pointed out, the application of noise filtering in addition to inverse filtering may also provide nonlinear corrections of the digital filtering results. To filter single photon counting measurements, a Fourier-spectrum continuation has been developed taking into account errors form a Poissonian distribution. ${ }^{30}$ Another method is based on an evenly distributed error (white noise) in the entire frequency domain and uses an adaptive Wiener filter. ${ }^{31,32}$ Adaptive filtering is a promising field to harness inverse filtering to get reasonably noiseless deconvolved ultrafast kinetic datasets without spurious fluctuations. Regularization is another possibility to "force" the inverse filtered object function to have some prescribed properties. ${ }^{33,34}$

It is worth mentioning a comparative study of six different deconvolution techniques by Madden et al. ${ }^{35}$ where the reader can find a numerical analysis of some specific methods used to deconvolve pharmacokinetic drug response functions. However, these methods apply either an implicit model function (e.g., cubic splines) or an interpolation of the original experimental data to a substantially increased grid size compared to the number of measured points. Though there are several distinct curve shapes considered, all of them are periodical starting from and ending at zero values. Consequently, results of this study do not have a great relevance to the deconvolution of ultrafast laser kinetics data.

\section{Numerical Tests of the Implemented Deconvolution Methods}

After having tried several deconvolution procedures, we have chosen to thoroughly test the applicability of those that could be successfully used in the deconvolution of femtosecond kinetic data. For the test purposes, we have used synthetic data, calculated on the basis of the simple consecutive mechanism

$$
\mathrm{A} \stackrel{\tau_{1}}{\longrightarrow} \mathrm{B} \stackrel{\tau_{2}}{\longrightarrow} \mathrm{C}
$$

with the initial conditions $[\mathrm{A}]=1 \mathrm{~mol} / \mathrm{dm}^{3},[\mathrm{~B}]=[\mathrm{C}]=0$ at $t=0$. The resulting kinetic response function is given in eq
(16) of ref 9. We have calculated three different kinetic curves (representing three detection wavelengths with different $\epsilon$ values of the individual species). The time series of $\Delta \mathrm{OD}$ values obtained this way were convolved with a $255 \mathrm{fs}$ fwhm Gaussian spread function and sampled at 30 fs intervals. To mimic experimental error, a random number was added to each sampled value, generated with a normal distribution. The mean of the distribution was set at zero and its variance at $2 \%$ of the maximum of the convolved data set, which is equivalent to an RMS error of $-34 \mathrm{~dB}$. The resulting raw data can be seen in Figure 1 of ref 10 . They were used as the input $i^{1}, i^{2}$, and $i^{3}$ with each deconvolution method, along with the error-free spread function sampled at the same time intervals.

Iterative model-free deconvolution methods were implemented as given above. The van Cittert linear iterative method has been tested only for comparison to see the degree of improvement when switching from a linear to a nonlinear iteration. As zeroth approximation we used $\hat{o}_{n}^{(0)}=i_{n}$, the convolved (image) data set. Iterative Bayesian deconvolution is computationally more demanding due to the double convolution included in each iteration step. As this method is sensitive to zero or near-zero values in the initial data set $i_{n}$, a "baseline correction" is needed if there is bleaching present or if experimental noise results in negative values of the $i_{n}$ set. This can be done by adding a positive constant $a$ to $i_{n}$ so that $\left(i_{n}+\right.$ $a)>0$ for all $n$. Once the Bayesian deconvolution is done, the same constant can be subtracted from the resulting $\hat{o}_{n}$ data. Obviously, this treatment introduces some distortion of deconvolution results. Jansson's method has been used with the relaxation function given in eq (30), where there is a need for the minimum and maximum of the true solution $o_{n}$, which is not easy to know prior to deconvolution. Even in the case of no bleaching or fluorescence detection, only the zero minimum is known, not the maximum. To get the extrema of the true object function, we made a Bayesian deconvolution where you do not need those two parameters, then started a new Jansson deconvolution using the upper and lower boundary limits thus obtained. Gold's ratio method should certainly provide more noisy results than the Bayesian method that has an additional smoothing in each iteration step. It was included in the present study to show exactly this improvement when the computationally more demanding Bayesian method is used.

Inverse filtering methods are based on Fourier and inverse Fourier transforms. Though the popular FFT method is also appropriate to use when calculating forward or inverse transforms, we usually get better results with the direct discrete Fourier transform (DFT) and its inverse transform if the number of data $N$ does not match exactly a power of 2 . This is probably due to the fact that padding the dataset with zeros (or other arbitrary values) to $N=2^{k}$ when using FFT usually results in a distortion of the transform. With recent computers, DFT calculations up to a few hundred data can be carried out within reasonable time. Implementing the DFT algorithm, we applied no normalization in the forward, and a normalization by $N$ (the length of the data set) in the inverse transformation, as given in eqs (5) and (6) in ref 10.

As we have pointed out before, simple inverse filtering enormously amplifies high-frequency noise, so this method cannot be applied without effectively filtering out this noise. We have tried four different filtering methods. One was prefiltering the $i(t)$ dataset using the reblurring procedure described above. Another method was the use of a simple lowpass filter, i.e., cutting the Fourier transform $\hat{O}$ above a threshold frequency $f_{\mathrm{o}}$ prior to inverse transformation. Neither of these 
filters gave satisfactory results. A Wiener filter is more sophisticated, which minimizes the sum of squared differences between the original function $f$ and the inverse Fourier transform of $\hat{F}$, denoted by $\hat{f}$. As the original $f$ function is not known, approximations for the optimal Wiener filter are usually used. A critical study of different formulations of the Wiener filter and their applicability to radioactive indicator-dilution data is described by Bates. ${ }^{32}$ In the case of a white noise, where the noise amplitude is the same constant $N$ at each frequency, the Wiener filter can be implemented in the following approximated form: ${ }^{9,31}$

$$
\hat{O}=\frac{|I|^{2}}{|I|^{2}+\frac{|N|^{2}}{|S|^{2}}} I \frac{1}{S}
$$

This filter, called adaptive pseudo-Wiener filter, has been successfully used to deconvolve radioactive indicator-dilution response curves by Gobbel and Fike. ${ }^{31}$ Finally, we also tried the two-parameter regularization filter proposed by Dabóczi and Kollár. ${ }^{34}$ Its simpler versions with only one additive constant in the denominator (the equivalent of $\lambda$ ) are widely used, as was proposed, e.g., by Parruck et al. ${ }^{36}$ Implementation of this filter was similar to that of the Wiener filter; the inverse filtered result was multiplied by this filter, giving the Fourier transform of the object as

$$
\hat{O}=\frac{I S^{*}}{|S|^{2}+\lambda+\gamma|L|^{2}}
$$

Here,

$$
|L(v)|^{2}=16 \sin ^{4}\left(\frac{\pi v}{v_{\mathrm{s}}}\right)
$$

is the square of the absolute value of the Fourier transform of the second-order backward differential operator, $S^{*}$ means the complex conjugate of the frequency-domain function $S$, and $v_{\mathrm{s}}$ is the sampling frequency, which can be calculated from the equidistant sampling time $\Delta t$ as $1 / \Delta t$. This filtering avoids division by zero where $|S|^{2}$ becomes practically zero, with the addition of the constant $\lambda$ and the frequency-dependent correction $\gamma|L|^{2}$ to the denominator. Regularization has been applied to isothermal DSC data by Pananakis and Abel, ${ }^{29}$ using the equivalent of eq (35) with $\gamma=0$, i.e., a one-parameter only regularization filter.

It is interesting to compare the last two filters. For a better comparison, let us multiply both the numerator and denominator of eq (34) by $S * /|I|^{2}$ to get

$$
\hat{O}=\frac{I S^{*}}{|S|^{2}+\frac{|N|^{2}}{|I|^{2}}}
$$

Comparing eqs (35) and (37) we see that singularities in both filters are avoided by additional terms to $|S|^{2}$ in the denominator, thus providing regularization of the deconvolution. In this respect, Wiener filtering can also be considered as a special case of regularization. However, the three different additive terms have different "side effects" in addition to avoiding singularities. The constant $\lambda$ smoothes also the signal itself, thus reducing eventual experimental noise independently of the frequency. The role of the term $|N|^{2} /|I|^{2}$ in the Wiener filter is also to reduce experimental noise, but the effect of the constant error power $|N|^{2}$ is modified when divided by the frequencydependent image power $|I|^{2}$. The smaller the power of the image (i.e., the higher the frequency), the greater the smoothing effect as well, in addition to the regularizing effect. The term $\gamma|L|^{2}$ in the regularization filter is similar in that its smoothing effect increases with increasing frequency, but in this case independently of the image power.

There is an additional problem with the Fourier transformation of femtosecond kinetic data: many measured datasets are nonperiodic, as can be seen from Figures 2, 4, and 7. This nonperiodic nature makes the Fourier transforms have virtual high-frequency components, as the difference from zero at the end of the data sets means a discontinuity in a circular transformation, which generates high-frequency components characteristic of steplike functions. These extra frequencies further increase spurious fluctuations in the deconvolved result, so they should be treated prior to deconvolution. There are methods described in the literature to avoid this problem in different ways. We have used the method proposed by Gans and Nahman ${ }^{37}$ by subtracting the shifted data from the original dataset to give a strictly periodic, finite support sequence. This results in a well-behaved Fourier transform, but provides twice the number of data in the frequency domain. However, due to double sampling of the time-domain data, every other item in the frequency-domain dataset is zero, which can be omitted if we plot an amplitude spectrum. As a result, we get the discrete Fourier transform of the unmodified dataset but without the highfrequency components that would appear if not performing the transformation to a periodic sequence. It should be noted that other proposed methods to treat steplike functions prior to Fourier transformation are equivalent to the Gans-Nahman method. ${ }^{38}$

The object functions $\hat{o}_{n}$ obtained with each deconvolution method mentioned above were analyzed the following way. Using the least-squares iterative parameter estimation of Marquardt $^{39}$ for all three datasets simultaneously, the parameters of the model function and their standard deviations were determined. On the basis of these values, the $95 \%$ confidence interval was calculated for each parameter. ${ }^{11}$ Confidence intervals calculated this way were compared to the least-squares iterative reconvolution results obtained from the $i(t)$ data with the convolved model function. As the reconvolution procedure contained all information except for the parameters concerning the object function during deconvolution, parameters obtained this way represent the best available estimates. In addition, various overall statistics and Fourier transform properties were also calculated. Fourier transforms can help to check the noise content of the measured data and follow the extent of noise reduction during deconvolution. To this end, we show some amplitude spectra in the frequency domain of the measured and restored datasets.

As for testing purposes we use synthetic data, so we are in a position to calculate the mean square error (MSE) of the deconvolution for the object function defined as

$$
\mathrm{MSE}_{\text {object }}=\sqrt{\frac{\sum_{m=i}^{N}\left(\hat{o}_{m}-\mathrm{o}_{m}\right)^{2}}{N-1}}
$$

We also calculate a similar statistics using the sum of squares of differences between the reconvolved results and the original 

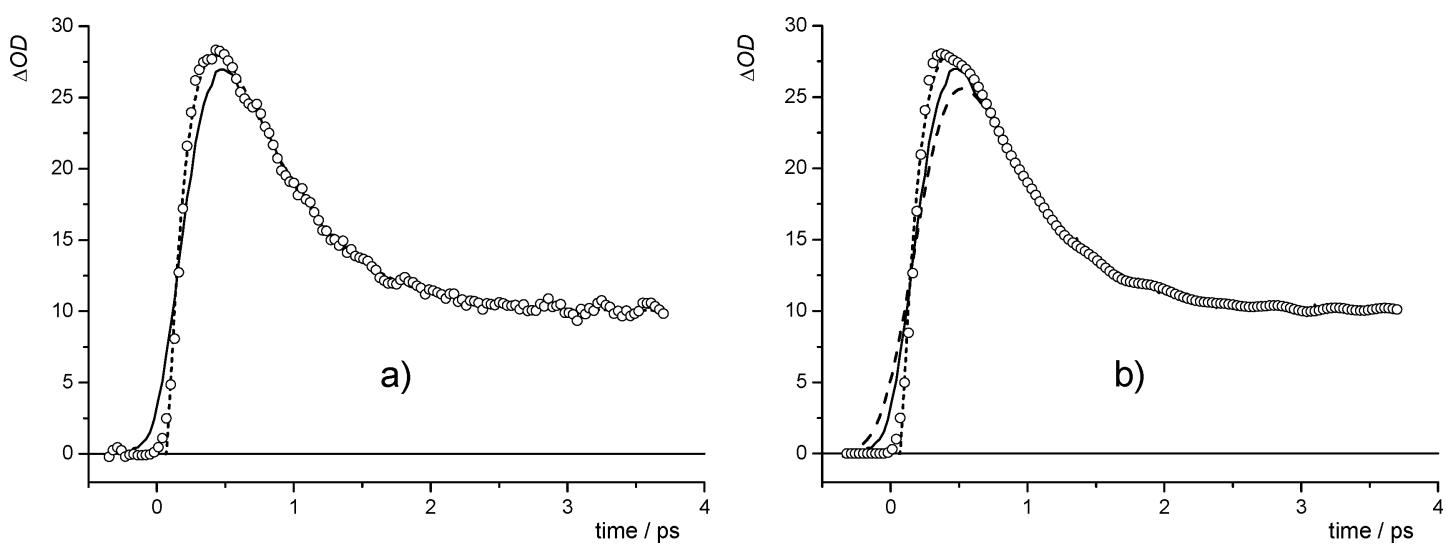

Figure 2. Datasets obtained with optimal Bayesian deconvolution from the simulated measured data (image) $i^{2}$. (a) Deconvolution of the original image. (b) Deconvolution of the reblurred image. Circles are the results of the deconvolution. The continuous curves show the image, and shortdashed curves indicate the original (noise-free) object function, while the long-dashed curve in panel $b$ shows the reblurred image. Note the noisereducing effect of the reblurring procedure.

image function defined as

$$
\mathrm{MSE}_{\text {image }}=\sqrt{\frac{\sum_{m=1}^{N}\left[(\hat{o} \otimes s)_{m}-i_{m}\right]^{2}}{N-1}}
$$

This latter can be calculated also in case of a measured $i_{m}$ dataset, when we do not know the true object function. Another indication of the quality of the deconvolved data $o_{m}$ is the oscillation index proposed by Gobbel and Fike. ${ }^{9,31}$ This index shows the extra oscillation with respect to a smooth unimodal function increasing monotonically from $\hat{o}_{1}$ to $\hat{o}_{\text {peak }}$, and decreasing monotonically from $\hat{o}_{\text {peak }}$ to $\hat{o}_{N}$.

(a) Test Results. When testing iterative methods, we monitored the mean-square errors $\mathrm{MSE}_{\text {object }}$ and $\mathrm{MSE}_{\text {image }}$ and the oscillation index OSC and fitted the model function to the deconvolved curves after each iteration step. The minimum of the mean-square errors or the minimal residual error in the fit was considered as an optimum for the deconvolution. When using the van Cittert or Gold methods, after a slight decrease during two to four steps, there was a monotonic increase of the errors indicating an ever increasing noise in the deconvolved data sets, while there were marked minima in the case of the Jansson and the Bayesian methods of $\mathrm{MSE}_{\text {object }}$ and the residual error in the fit. Though the two minima usually did not coincide, they did not differ much either. (As an example, it was at 58 iteration steps for $\mathrm{MSE}_{\mathrm{object}}$ and 63 steps for the residual error in the fit when deconvolving the function $i^{2}$ using the Bayesian method. Cf. Figure 3.) However, there was not any minimum in either the $\mathrm{MSE}_{\text {image }}$ error or the oscillation index OSC during iteration with any methods that would have provided reasonable deconvolved data. In some cases, there appeared extrema in OSC but the minima never indicated reasonable deconvolution results either, and occurred prior to the optimal iteration number found based on the $\mathrm{MSE}_{\text {object }}$ or residual fit. Fitted parameters from the Bayes-deconvolved data set were quite reliable, while those from the Jansson-deconvolved data set usually had too large errors.

When deconvolving reblurred data sets, there were always minima for the $\mathrm{MSE}_{\text {object }}$ and the residual error in the fit, and results obtained for fitted parameters were comparable in case of all the methods. Both OSC and $\mathrm{MSE}_{\text {image }}$ have shown a monotonic change with iteration number, so they cannot be used as optimum criteria. Considering all the parameters, it was also the Bayesian method which gave the best result. Table 1A)

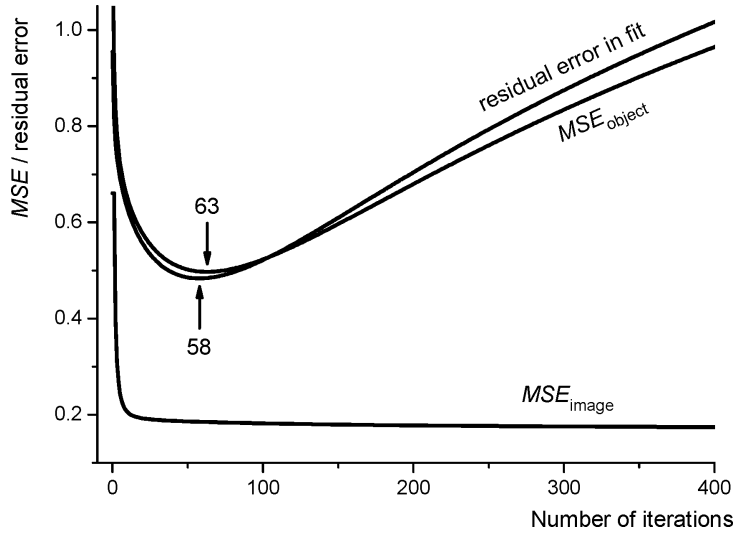

Figure 3. Different optimum criteria as a function of the number of iterations for a Bayesian deconvolution of the original $i^{2}$ dataset. Arrows with iteration numbers indicate the corresponding minima. Note the monotonic decrease of $\mathrm{MSE}_{\text {image }}$ with the iteration number.

summarizes parameters thus obtained from the fit. As can be seen from the table, the best-performing method is Bayesian iteration of the reblurred image data.

To show the quality of deconvolution, two examples can be seen in Figure 2. Figure 3 shows the existence of optima during the iterative procedure. The oscillation index OSC of the deconvolved data increased monotonically (not shown in Figure 3 ), due to the monotonically increasing amplified noise.

Testing inverse filtering methods, we have used a similar optimization strategy as with time-domain iterative methods. The only difference was that, in this case, OSC, MSE $\mathrm{object}_{\text {, }}$ $\mathrm{MSE}_{\text {image }}$, and the residual error in the fit were monitored as a function of the filter parameter(s). According to our results, preliminary noise filtering using reblurring does not help to eliminate the enormous noise amplification during the inverse filtering procedure if we do not apply additional filters. Though the simple low-pass filter helps reducing this noise to a large degree, it still introduces relatively large spurious fluctuations in the obtained deconvolved data set while still heavily distorting the deconvolved signal shape. The Wiener filter and regularization result in acceptable deconvolved data sets, so we explored in detail only the use of these additional filters. The relevant parameter for the Wiener filter (eq (34)) is the noise power $|N|^{2}$, while for the regularization filter, there are two parameters, $\gamma$ and $\lambda$.

Before searching for optima in the two-parameter space, we have used first a one-dimensional search also for the regularization filter, setting either $\gamma$ or $\lambda$ to zero. The upper limits of a 
TABLE 1: Estimated Parameters Obtained When Fitting Model Function (33) to the Three Simulated Datasets Deconvolved Using Different Deconvolution Methods and the Residual Error in the Fit as Optimum Criterion ${ }^{a}$

(A) Iterative Deconvolution in Time Domain

\begin{tabular}{|c|c|c|c|c|c|c|}
\hline parameters & $\begin{array}{l}\text { true } \\
\text { value }\end{array}$ & Bayesian & $\begin{array}{l}\text { Bayesian } \\
\text { reblurred }\end{array}$ & $\begin{array}{l}\text { van Cittert } \\
\text { reblurred }\end{array}$ & $\begin{array}{l}\text { Gold } \\
\text { reblurred }\end{array}$ & $\begin{array}{l}\text { Jansson } \\
\text { reblurred }\end{array}$ \\
\hline$\tau_{1}$ & 0.20 & $0.19(0.02)$ & $0.19(0.02)$ & $0.22(0.04)$ & $0.20(0.03)$ & $0.20(0.03)$ \\
\hline$\tau_{1}$ & 0.50 & $0.49(0.04)$ & $0.50(0.04)$ & $0.46(0.06)$ & $0.48(0.04)$ & $0.49(0.04)$ \\
\hline$\epsilon_{\mathrm{A}}^{1}$ & 30 & $28.9(1.4)$ & $29.4(1.3)$ & $26.4(1.5)$ & $28.0(1.4)$ & $28.0(1.4)$ \\
\hline$\epsilon_{\mathrm{B}}^{\mathrm{A}}$ & 20 & $19.6(1.3)$ & $19.2(1.2)$ & $20.1(1.7)$ & $19.7(1.4)$ & $19.8(1.4)$ \\
\hline$\epsilon_{\mathrm{A}}^{2}$ & 5 & $3.5(1.6)$ & $3.5(1.5)$ & $5.0(1.8)$ & $4.0(1.5)$ & $3.2(1.6)$ \\
\hline$\epsilon_{\mathrm{B}}^{2}$ & 45 & $45.7(2.7)$ & $45.2(2.5)$ & $47.9(4.5)$ & $46.5(3.1)$ & $45.9(2.9)$ \\
\hline$\epsilon_{\mathrm{C}}^{2}$ & 10 & $10.0(0.3)$ & $10.0(0.3)$ & $10.1(0.3)$ & $10.1(0.3)$ & $10.0(0.3)$ \\
\hline$\epsilon_{\mathrm{A}}^{3}$ & 5 & $4.2(1.4)$ & $4.2(1.4)$ & 4.8 (1.6) & $4.5(1.4)$ & $5.2(1.5)$ \\
\hline$\epsilon_{\mathrm{B}}^{3}$ & 30 & $30.5(2.1)$ & 30.2 & $32.1(3.3)$ & $31.1(2.3)$ & $30.1(2.2)$ \\
\hline$\epsilon_{\mathrm{C}}^{3}$ & -10 & $-9.9(0.3)$ & $-9.9(0.3)$ & $-9.8(0.4)$ & $-9.8(0.3)$ & $-9.9(0.3)$ \\
\hline
\end{tabular}

(B) Inverse Filtering in Frequency Domain

\begin{tabular}{|c|c|c|c|c|}
\hline \multirow[b]{2}{*}{ parameters } & \multirow{2}{*}{$\begin{array}{c}\text { true } \\
\text { value }\end{array}$} & \multirow{2}{*}{$\begin{array}{l}\text { Wiener } \\
\text { filter }\end{array}$} & \multicolumn{2}{|c|}{ regularization } \\
\hline & & & optimized $\gamma$ & optimized $\lambda$ \\
\hline$\tau_{1}$ & 0.20 & $0.22(0.04)$ & $0.23(0.04)$ & $0.24(0.05)$ \\
\hline$\tau_{2}$ & 0.50 & $0.45(0.06)$ & $0.45(0.06)$ & $0.44(0.07)$ \\
\hline$\epsilon_{\mathrm{A}}^{1}$ & 30 & $26.5(1.5)$ & $26.2(1.5)$ & $25.7(1.5)$ \\
\hline$\epsilon_{\mathrm{B}}^{1}$ & 20 & $20.1(1.7)$ & $20.2(1.7)$ & $20.1(1.8)$ \\
\hline$\epsilon_{\mathrm{A}}^{\mathrm{D}}$ & 5 & $4.9(1.8)$ & $5.0(1.8)$ & $5.2(1.7)$ \\
\hline$\epsilon_{\mathrm{B}}^{2}$ & 45 & $48.0(4.6)$ & $48.3(4.8)$ & $48.0(5.4)$ \\
\hline$\epsilon_{\mathrm{C}}^{2}$ & 10 & $10.1(0.3)$ & $10.1(0.3)$ & $9.9(0.3)$ \\
\hline$\epsilon_{\mathrm{A}}^{3}$ & 5 & $4.7(1.7)$ & $4.8(1.6)$ & $4.8(1.6)$ \\
\hline$\epsilon_{\mathrm{B}}^{3}$ & 30 & $32.1(3.4)$ & $32.4(3.5)$ & $32.2(3.9)$ \\
\hline$\epsilon_{\mathrm{C}}^{3}$ & -10 & $-9.8(0.4)$ & $-9.8(0.4)$ & $-9.6(0.4)$ \\
\hline
\end{tabular}

${ }^{a}$ Characteristic times $\tau_{1}$ and $\tau_{2}$ are given in ps units, while molar absorptivities $\epsilon_{i}^{\lambda}$ are given in $\mathrm{dm}^{3} \mathrm{~mol}^{-1} \mathrm{~cm}^{-1}$. Numbers in italics indicate a systematic error in estimation. Bold nonitalic numbers are the best estimates. Bold italics indicate the best estimate but with a systematic error. Headings indicate the actual deconvolution method. Numbers in parentheses show the half-widths of $95 \%$ confidence intervals. Note that $\epsilon_{\mathrm{C}}^{1}$ was set to zero and not treated as a fitted parameter.

subsequent $100 \times 100$ division two-dimensional grid search were set to be somewhat higher as the optimal values obtained in one-parameter optimizations, while the lower limits were set to zero. For the test functions studied here, we have found an interesting result: in all of the two-parameter optimizations, one of the parameters was found to be zero at the optimum. Obviously, the other parameter was equal to the value obtained from the one-parameter optimization, so the two-parameter regularization can be reduced to one-parameter regularization in the case of the studied model functions. In the case of function $i^{1}$, two-parameter optimization was found at nonzero $\lambda$ parameter, with $\gamma=0$, while with the functions $i^{2}$ and $i^{3}$ we have got the same optimal $\gamma$ value as in the case of the one-parameter $\gamma$-optimization, with $\lambda=0$. Consequently, only test results obtained from one-parameter optimizations are given in Table 1B. Since the more demanding two-parameter regularization did not provide better results with respect to the one-parameter regularization, there is probably no need to use it in the case of image functions similar to those tested here.

Tracing the results of the grid search, there were marked minima of $\mathrm{MSE}_{\text {object }}$ and the residual error in the fit, which gave only slightly different optimal values. However, there was not any minimum in either the $\mathrm{MSE}_{\text {image }}$ error or the oscillation index during the optimization with any methods. After a sharp initial decrease, the OSC curve shows a very shallow but monotonic decrease with increasing filter parameter (cf. Figures 2 and 3 of ref 9.), while the MSE $E_{\text {image }}$ error constantly increases.

It is interesting to follow the Fourier transforms of different data sets during the optimization procedure. When applying only the simple inverse filter with no additional noise filtering (i.e., $\gamma, \lambda$, or $|N|^{2}$ parameters are set to zero), after a moderate initial amplification of the signal at low frequencies, the experimental noise becomes gradually more and more overamplified so that high frequencies at the end of the spectrum have typically several thousand times greater amplitudes than in the original undistorted signal. (Obviously, this leads to a time domain data set with several orders of magnitude higher noise than the signal itself.) As the relevant filter parameters are increased, this highfrequency noise is gradually damped by the additional filter, while the low-frequency amplification remains practically the same. The optimal filter parameter is that which effectively damps the high-frequency noise with no important distortion of the low-frequency part, this latter containing most of the information of the useful time-domain signal. To demonstrate this effect of the additional noise filter, we show a few spectra at optimal noise filter parameters.

In Figure $4 b$, we can see the frequency characteristics of different filters resulting in the deconvolved time-domain signals shown in panel a. At about $2.5 \mathrm{THz}$, the Wiener filter has a sharp cutoff that reaches its highest value of about 20 thousand time damping at about $4.5 \mathrm{THz}$ (apart from fluctuations), which remains more or less constant till the end of the spectrum at about $16 \mathrm{THz}$. Both $\gamma$ and $\lambda$ regularization filters have less sharp and higher frequency cutoff characteristics; however, the frequency-dependent $\gamma$-filter has a steeper breakdown than the frequency-independent $\lambda$-filter, so it gradually catches up with the Wiener filter curve to cut somewhat more from about $4 \mathrm{THz}$. From here on, the damping of the Wiener filter is oscillating 

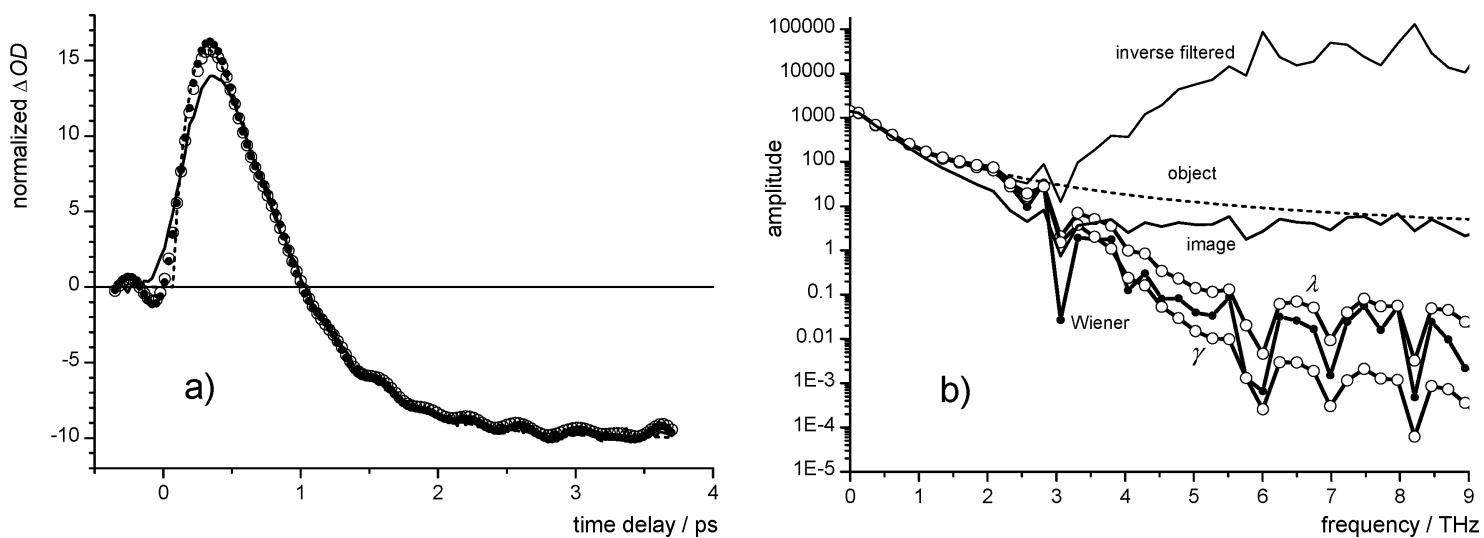

Figure 4. (a) Deconvolved data sets obtained with different inverse filters from the simulated measured data set (image) $i^{3}$. Open circles show the results obtained with optimized regularization filters $(\lambda$ - and $\gamma$-filtered results [at $\lambda=0.0179$ and $\gamma=0.244$, respectively] are not discernible visually at this scale). Smaller filled circles show the results obtained with an optimal Wiener filter [at $|N|^{2}=1.24 \times 10^{-5}$ ]. The continuous curve shows the image, and the short-dashed curve indicates the original (noise-free) object function. (b) Amplitude spectra of the deconvolved data sets in panel a. Circles show spectra of the deconvolved data sets, each one marked with the corresponding filter name. The top thin solid curve shows, for comparison, the spectrum of the simple inverse filtered result without any noise removal.
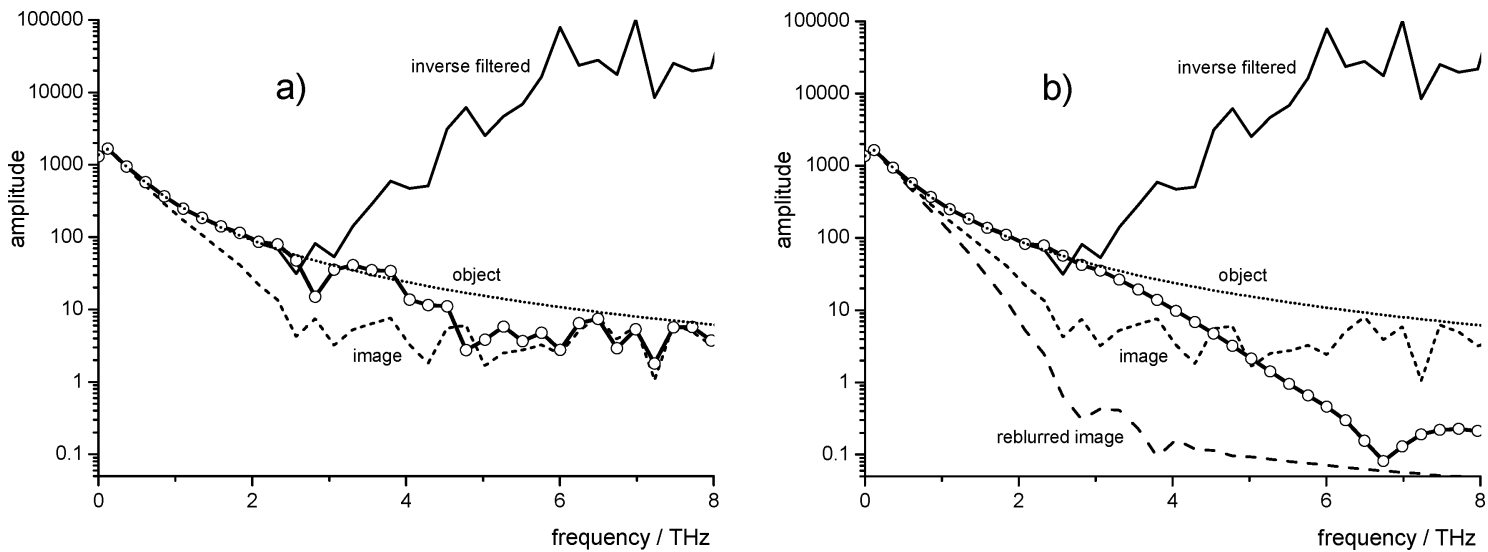

Figure 5. Amplitude spectra of the deconvolved data sets obtained with Bayesian deconvolution from the simulated measured data set $i^{2}$ (see time domain results in Figure 2). (a) Deconvolution of the original image. (b) Deconvolution of the reblurred image. Circles show spectra of the deconvolved data sets, dotted curves indicate the original (noise-free) object function, and short-dashed curves indicate the measured signal, while the longdashed curve in panel $b$ shows the spectrum of the reblurred image. The top thin solid curve shows, for comparison, the spectrum of the simple inverse filtered result without any noise removal. Note the noise-reducing effect of the reblurring procedure.

between that of the $\lambda$-filter and that of the $\gamma$-filter, depending on the actual noise of the measured data. Summing up we may say that the Wiener filter reduces the low-frequency noise more efficiently, and its damping at higher frequencies seems to "imitate" an optimum between the $\lambda$ - and the $\gamma$-filter, depending on the experimental noise level. This behavior of the frequency spectra is quite similar for the deconvolution results of the other two data sets $i^{1}$ and $i^{2}$.

Model parameters obtained when estimating from the inverse filtered deconvolved data sets are shown in Table 1B. It can be seen that the errors of the characteristic times are greater than those obtained from the Bayesian iteration results, but many absorptivity parameter errors are comparable to those obtained from iterative results. The overall performance of the Wiener filter is the best, though there is not much difference between regularization filtered and Wiener filtered results in the time domain, as it is expected from the above considerations of the frequency spectra.

The frequency-domain behavior suggests a similar analysis of the time-domain iteration results as well. As an example, in Figure 5 we show amplitude spectra of the Bayesian deconvolution results obtained from the image function $i^{2}$ (cf. Figure 2 to see corresponding time-domain results). It is worth noting the minimal suppression of the high frequencies in panel a, in the case of the Bayesian deconvolution of the original (nonre- blurred) image data. Obviously, the slight overamplification between 3 and $4 \mathrm{THz}$ is responsible for the minor extra oscillation at the end of the deconvolved curve in Figure 2a, but the reason for keeping the experimental noise of the image in the deconvolved data set is also this very small noise suppression. In panel $b$ of Figure 5 we see an important cut starting at $\sim 3 \mathrm{THz}$ and increasing down to more than a 100 times damping of the high frequencies. This frequency behavior is responsible for the efficient filtering of the experimental noise in the resulting time-domain data set (cf. Figure $2 b$ ).

Frequency characteristics of the van Cittert, Gold, and Jansson iterative deconvolution results show that the high-frequency damping effect is substantially less if using these methods than with the Bayesian deconvolution. It is not surprising that the Jansson method works well in spectroscopy and chromatography; it enhances efficiently the high-frequency components as the iteration proceeds, thus efficiently increasing even small peaks. However, most of the femtosecond kinetic data do not contain several small peaks, rather one shallower "bump", so the gradual amplification of smaller features by the Jansson method is not desirable in this case, but it is responsible for the greater errors obtained for parameters estimated from the Jansson-deconvolved data. Even so, Jansson deconvolution can be useful if there is a need for a steep initial rise but also for an efficient suppression of too high a peak resulting from this rise, 

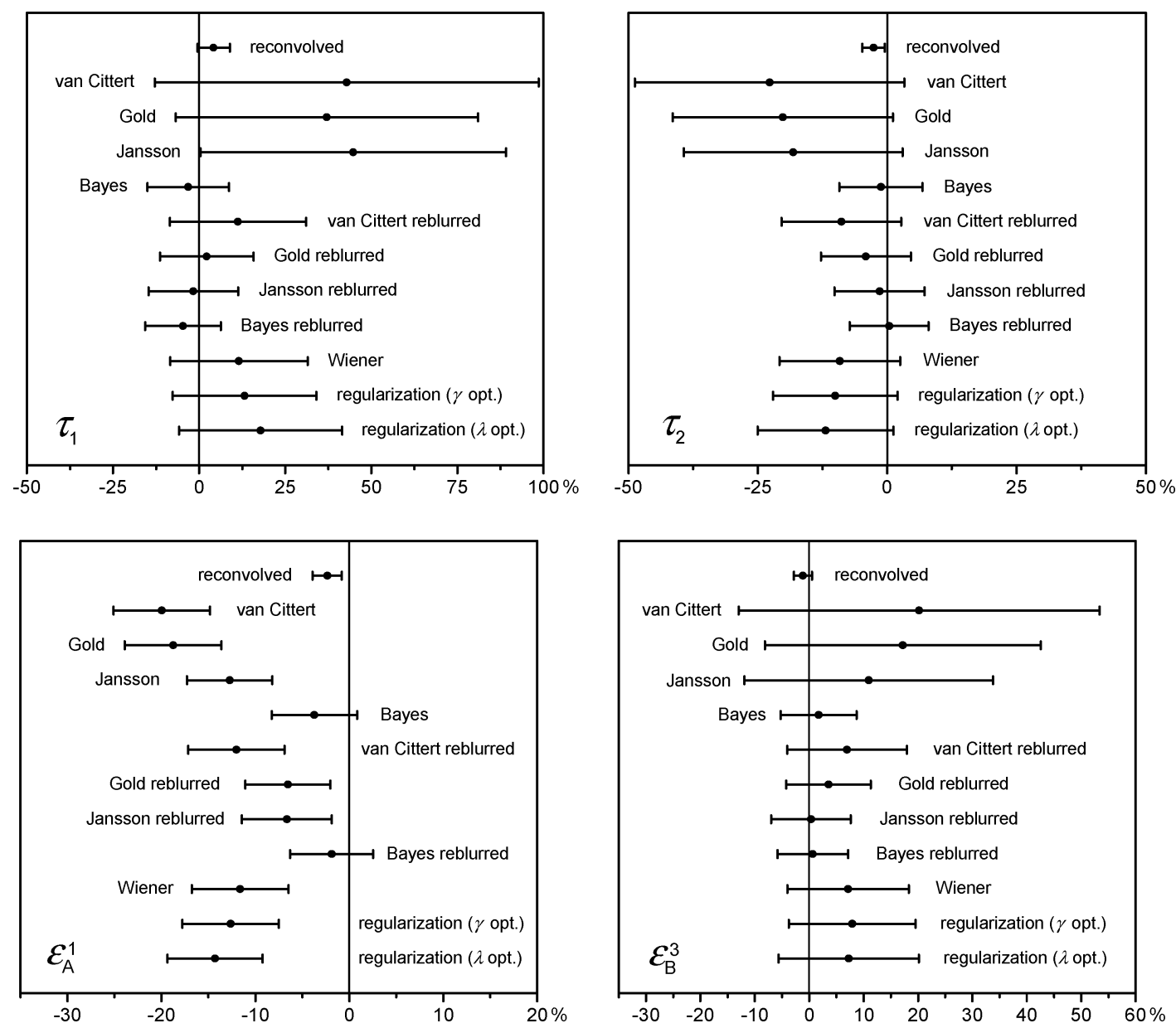

Figure 6. Upper two panels: $95 \%$ confidence intervals in percentage relative to the parameter value (relative errors) for the two time constants $\tau_{1}$ and $\tau_{2}$ in eq (33) obtained from a global fit to three deconvolved data sets resulting from different deconvolution methods. Methods are identified by the text written aside the corresponding error bars. The top bar in each frame marked "reconvolved" was obtained with a reconvolution procedure, representing the estimate obtained using the proper model function. Lower two panels: Relative errors for two molar absorptivity coefficients. $\epsilon_{\mathrm{A}}^{1}$ (reactant) was obtained with the greatest, while $\epsilon_{\mathrm{B}}^{3}$ (transient) with the smallest systematic error.

as this method efficiently avoids a rise above the prescribed maximum. Reshaping the original triangular relaxation function can also help to get more suitable deconvolution results with the Jansson method (see e.g. figure 8).

(b) Recommendations. On the basis of the test results described above, we can derive some principles to be followed when trying to deconvolve femtosecond kinetic data without any chemical and/or photophysical model. To help the comparison of different methods, we visualize in Figure 6 some results obtained with different deconvolution methods tested, showing estimated parameter values (central dots) and their relative errors (bars) as $95 \%$ confidence intervals in percentage of the respective estimated values. The top bar is the result obtained from reconvolution, i.e., when fitting the known model function (33), convolved with the known effective pulse, to the image data. This result represents the best available estimate of the parameters, as the fitting procedure makes use of the complete knowledge of the true model, except for the actual values of its parameters. The vertical lines in the diagrams indicate zero error, i.e., no systematic distortion of the parameter. From the data shown in Figure 6, we can easily see the statistical precision of the estimated parameters (bar size), and the systematic distortion (relative position of the dots and the bars with respect to the zero line).

A striking feature of the diagrams is that several methods, mostly time-domain iterations of the reblurred data sets, provide less systematic error than reconvolution. This is due to the fact that a nonparametric deconvolution results in a deconvolved data set obtained independently from the kinetic/photophysical model, thus the "fine-tuning" of the pulse parameters (zero time and exact width) does not correlate with the model parameters. Bayesian deconvolution of the reblurred data gives the most remarkable results in this respect; parameters obtained from these data are always much less distorted than those from the reconvolved results. It should also be noted that the statistical error is of course greater (about twice as large) with respect to the reconvolution results, but this is normal as the information content of the true model is not used at all when deconvolving without any model. However, the systematic error of the estimated value (the distance of the central dot from zero) is often less than that of the reconvolved result, and never really greater. Bayes deconvolution (both of the original and the reblurred image) gives a nondistorted result even for the most distorted parameter $\epsilon_{\mathrm{A}}^{1}$, where all other methods fail, including reconvolution.

On the basis of these findings we can recommend a nonparametric deconvolution and subsequent parameter estimation from fitting the model function to the deconvolved data set even in the case if the model function is known from other experimental evidence than the ultrafast kinetic measurements used to determine kinetic and photophysical parameters. Though statistical errors can be up to twice as much as estimated with a reconvolution procedure, markedly smaller systematic distor- 
tions can be expected with this method. If we are not certain of a physical model a priori and want to select the most suitable one based on the evaluation of the femtosecond kinetic data, nonparametric deconvolution efficiently helps to avoid unnecessary correlation of the fine-tuned pulse parameters with the model parameters, thus facilitating the choice between concurrent models.

Another interesting feature is that the results obtained with a Wiener noise filter are always very much the same as those obtained with the van Cittert iteration of the reblurred data. Both the van Cittert and the inverse filtering methods are linear deconvolution methods, so the explanation should be that reblurring in the time domain has probably the same noisereducing properties as a Wiener filter in the frequency domain. Observing the respective error bars, we can also state that the Wiener filter has somewhat less systematic distortion than the regularization filters. This observation supports that-if there is apparently no "fine structure" present in the measured data but it contains rather a simple "bump"-a Wiener noise filter is the method of choice if inverse filtering is used for deconvolution. However, if there are also smaller peaklike features to be suspected in the deconvolved curve, regularization, especially frequency-independent $\lambda$-regularization, might be a better choice, even if it provides somewhat larger statistical errors in the parameters. ${ }^{10}$

A similar recommendation can be formulated for the timedomain iterative methods. Bayesian deconvolution has by far the best statistical properties from the point of view of both a small systematic distortion and a narrow confidence interval due to the efficient smoothing effect of this method. However, if smaller peaks or a less smooth behavior with very steep a rise at early times are suspected, Jansson deconvolution of the reblurred image might give more satisfactory results. Van Cittert and Gold iterative methods do not have any advantages with respect to the above-mentioned two iterative methods, so their use in practical applications is not recommended. It should be noted that Bayesian deconvolution can only be applied to a nonnegative dataset, so the baseline-correction mentioned in describing the numerical implementation should be always used if necessary. Varying the baseline correction used, we have observed that the smallest distortion occurs if the smallest possible baseline correction is applied.

A few words should be added also on the optimization procedure if the methods are applied to real experimental data. In this case not only the model, but also the original, undistorted data set is unknown. While it is easy to use statistics derived from the undistorted signal to find optimal iteration numbers or filter parameters, when only the convolved image function measured in an experiment is known, there are no statistics available which would indicate optimal deconvolution. As we have shown in the previous chapter, both $\mathrm{MSE}_{\text {image }}$ and the oscillation index OSC have a monotonic behavior as a function of the relevant filter parameters. Though the existence of an optimum is well established on the basis of the results found for the case of known object functions, which certainly holds for experimental data of similar shape, there is no numerical optimum criterion if the true model function is unknown. However, on the basis of the frequency-domain characteristics of different data sets compared to the deconvolved data, a fairly good estimate of the optimal deconvolution can be chosen. As it can be seen in Figures 4 and 5, optimally deconvolved data have a frequency spectrum whose amplitude never turns from a decreasing tendency into an increase. This is supported by the figures showing deconvolution results of synthetic data, as

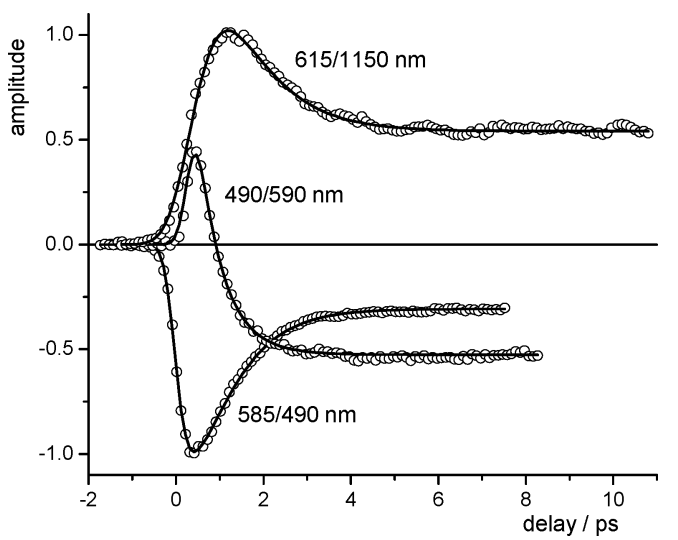

Figure 7. Experimental ultrafast kinetic curves deconvolved in this study, as measured by Barthel et al. ${ }^{40}$ Excitation/detection wavelengths shown identify individual curves. Circles denote measured data, and solid lines represent the best fit of the model as estimated by reconvolution from these three data sets. All data are normalized to unit amplitude between their highest and lowest values.

we can also plot the Fourier spectrum of the known undistorted function $o$. Whenever the amplitude of the deconvolved spectrum exceeds that of the monotonically decreasing undistorted function $o$, the excess amplitude only enhances noise in the deconvolved data set. On the basis of this fact we should check for the onset of increasing noise tendency even if the undistorted function $o$ cannot be seen on the spectra, and stop the iteration or the decrease of the filter parameter (see Figure 3 ). Properties of this graphically observed optimum are discussed in more detail in ref 10 .

A computer program is also available as Supporting Information to this paper which facilitates graphical deconvolution via inverse filtering. It shows both time-domain and frequencydomain behavior of the relevant functions while changing filter parameters. The effect of choosing a Fourier transform method in the case of steplike functions can also be observed using the program.

\section{Deconvolution of Real-Life Experimental Data}

Real experimental data might differ much from those calculated on the basis of the simple two-step model used to test the applicability of deconvolution procedures. We have chosen a considerably complex phenomenon, the CTTS (charge transfer to the solvent) of the sodide ion $\mathrm{Na}^{-}$observed in THF (tetrahydrofurane) solution to deconvolve with the abovedescribed nonparametric methods. The original data along with a reconvolution analysis using a quite sophisticated model is described at length in a previous paper. ${ }^{40}$ Figure 7 shows the three experimental curves chosen here to deconvolve, all normalized to unit amplitude between their highest and lowest values. The choice of three substantially different shapes makes it possible to estimate the parameters of the reported kinetic model, consequently, a comparison of the quality of inference from nonparametric deconvolution with respect to reconvolution. (Note that we have kept the parameters of the shifting spectrum of the neutral $\mathrm{Na}^{0}$ species, formed when the electron gets detached from the $\mathrm{Na}^{-}$ion, as published in ref 40 , for measured data at three wavelengths only are not sufficient to estimate them with a reasonable precision.)

We performed iterative Bayesian and Jansson deconvolution, and inverse filtering with additional Wiener or regularization noise filtering on all three curves. In addition to the near-optimal deconvolutions found using the above-described graphical observation of the noise behavior, we checked slightly under- 

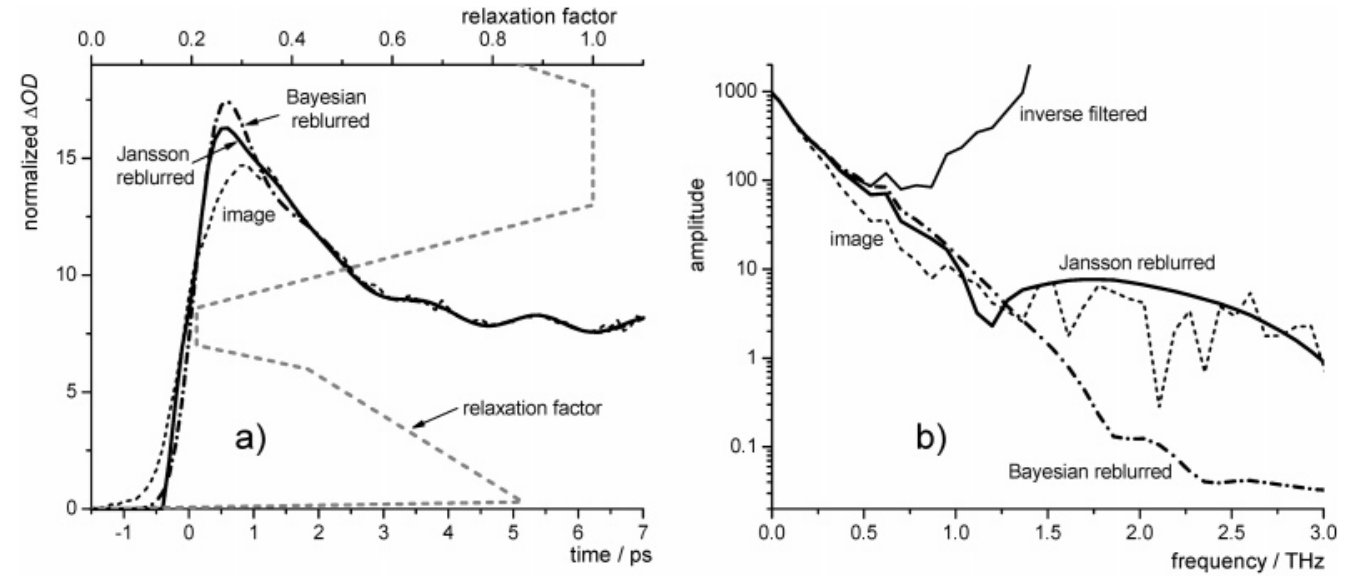

Figure 8. Experimental ultrafast kinetic curves detected at $1150 \mathrm{~nm}$, deconvolved with a Bayesian and a Jansson method of the reblurred data set. The double quadrangular shape of the relaxation function used with the Jansson method (long dashed gray curve) is also shown in panel a. Amplitude spectra of the respective curves are shown in panel $b$.

filtered and slightly overfiltered results as well, to see the quality of the chosen optima. This checking of the results was done by fitting the reported model function simultaneously to the three deconvolved data sets and comparing the statistics of the parameters estimated from the fit. We have used the Marquardt method of nonlinear estimation, ${ }^{39}$ similarly to the case of simulated data.

It was found in each case that the underfiltered (when performing time-domain iterative deconvolution, this corresponds to the case of too high iteration numbers) as well as the overfiltered object reconstructions gave statistical results inferior to those found optimal based on the graphical observation of the frequency-domain behavior. This is in accordance with the findings reported in ref 10 that graphical observation is a robust method of optimal deconvolution. Underfiltered data sets resulted in considerably larger errors of the parameters, while overfiltered data sets had usually worse statistics indicating systematic error in the fit.

However, there were differences in this case with respect to the simulated data. As it can be seen in Figure 8, Bayesian deconvolution results in a reconstructed object function that obviously does not have a steep enough initial rise. We have found that, with a suitable "shaping" of the relaxation function using Jansson deconvolution, we can achieve a more satisfactory result than with the Bayesian method. The relevant relaxation function of Figure 8 has a double quadrangular shape. We can see that there is no amplification (zero relaxation factor) at exactly zero signal amplitude, but it increases very sharply to become 0.86 already at $\triangle \mathrm{OD}=0.3$, resulting in a largely increasing amplification above but close to zero. This amplification should gradually decrease to fall off to a negligible size between 7 and 8.6, at the region of the fluctuating constant value of the residual absorbance, as this fluctuation should rather be suppressed, but by no means largely amplified. (The original trigonally shaped Jansson relaxation function would have a near maximal value exactly in this region, thus increasing the fluctuation in an undesired way.) Above the constant residual absorbance, especially near the peak of the curve, the deconvolved function should have an enhanced peak, so that the relaxation function should become again very large. Shaping the relaxation function this way, we have achieved a really steep rise after a smooth zero signal level, without the overshooting of the Bayesian deconvolution result, as it can be seen from the comparison in panel a of Figure 8. In panel b we can see the corresponding frequency spectra showing that the Jansson deconvolution results in a lower amplification than the Bayesian

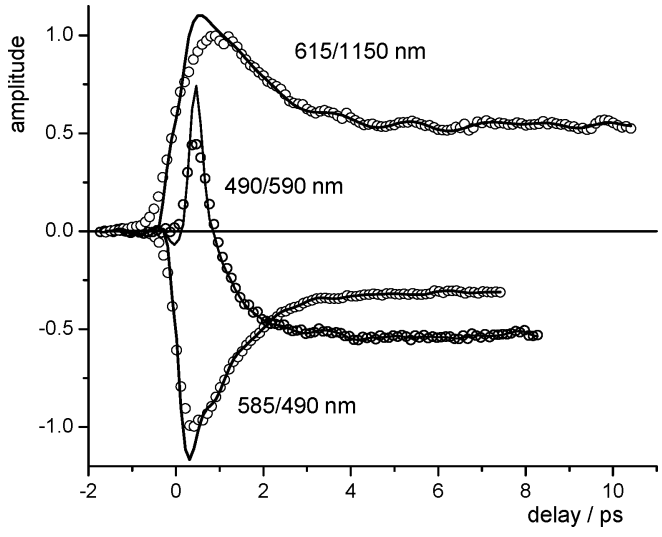

Figure 9. Deconvolved experimental data. Excitation/detection wavelengths shown identify individual curves (cf. Figure 7). Circles denote measured data; solid lines represent deconvolved data sets obtained with Jansson time-domain iterative deconvolution using relaxation functions similar to that of Figure 8. All data are normalized to unit amplitude between their highest and lowest values.

method in the low-frequency domain, avoiding unnecessary slow fluctuations as well as overshooting at the relatively shallow peak. At the same time, it allows higher amplitude at higher frequencies, thus providing the necessary steep rise at early times.

Inverse filtering also provides quite reliable results compared to that of the Bayesian or Jansson deconvolution. The quality of deconvolution with inverse filtering can be seen in Figure 4 of ref 10 . Though the results obtained with a Wiener filter are the best in statistical terms, $\gamma$-regularization is not much inferior. As in the case of the synthetic data set (see Figure 4 here, and Figures 2 and 3 of ref 10), inverse filtered curves also contain a low-frequency oscillation around the initial zero $\triangle O D$, but knowing that this oscillation is an artifact, we may only keep the monotonically rising part of the initial portion of the curve. However, this initial oscillation enables a considerably steeper rise than the flat initial part in the case of a Bayesian deconvolution. This is the reason for obtaining better results for the parameters than in the case of a Bayesian deconvolution, even if reblurring is applied.

Deconvolved data sets obtained using different methods, resulting in the best fit with the model function published in ref 40, are shown in Figure 9. Parameters obtained from the fits are shown in Table 2. To facilitate the comparison, Figure 10 shows four parameters obtained with different deconvolution 
TABLE 2: Estimated Parameters Obtained When Fitting the Model Function of Ref 40 to the Three Experimental Datasets Deconvolved Using Different Deconvolution Methods ${ }^{a}$

\begin{tabular}{lcclcrc}
\hline parameters & reconvolution & Bayesian & $\begin{array}{l}\text { Bayesian } \\
\text { reblurred }\end{array}$ & $\begin{array}{c}\text { Jansson } \\
\text { reblurred }\end{array}$ & $\begin{array}{c}\text { Wiener } \\
\text { filtered }\end{array}$ & $\begin{array}{c}\text { regularized } \\
\text { filtered }(\gamma)\end{array}$ \\
\hline$\tau_{1}$ & $0.79(0.06)$ & $\mathbf{0 . 7 8}(\mathbf{0 . 0 9})$ & $0.75(0.10)$ & $0.76(0.10)$ & $0.76(0.10)$ & $0.77(0.10)$ \\
$\tau_{2}$ & $0.53(0.05)$ & $0.33(0.05)$ & $0.35(0.06)$ & $\mathbf{0 . 3 8}(\mathbf{0 . 0 6})$ & $0.38(0.07)$ & $0.36(0.06)$ \\
$P_{585}$ & $0.80(0.01)$ & $0.91(0.01)$ & $0.91(0.02)$ & $0.90(0.02)$ & $\mathbf{0 . 8 9}(\mathbf{0 . 0 2})$ & $0.90(0.02)$ \\
$P_{490}$ & $0.55(0.09)$ & $0.64(0.13)$ & $0.58(0.17)$ & $0.58(0.15)$ & $\mathbf{0 . 5 6 ( 0 . 1 8 )}$ & $0.59(0.16)$ \\
$P_{615}$ & $0.77(0.02)$ & $0.84(0.03)$ & $0.82(0.03)$ & $\mathbf{0 . 8 1 ( 0 . 0 3 )}$ & $0.82(0.04)$ & $0.82(0.03)$ \\
$A_{585 / 490}$ & $15.4(1.2)$ & $27.5(0.5)$ & $27.3(0.6)$ & $26.3(0.7)$ & $\mathbf{2 6 . 1}(\mathbf{0 . 8})$ & $26.3(0.7)$ \\
$A_{490 / 590}$ & $70.8(5.1)$ & $62.3(5.8)$ & $64.8(7.8)$ & $\mathbf{6 4 . 8 ( 7 . 2 )}$ & $65.0(8.0)$ & $63.9(7.1)$
\end{tabular}

${ }^{a}$ Characteristic times $\tau_{1}$ and $\tau_{2}$ are given in ps units, while molar absorptivities $A_{\text {pump/probe }}$ are given in $\mathrm{dm}^{3} \mathrm{~mol}^{-1} \mathrm{~cm}^{-1}$. Parameters $P_{\text {pump }}$ stand for the branching ratios of electron detachment to form an immediate-rather then solvent separated-ion pair at the respective wavelength of the applied pump pulse. Headings indicate the actual deconvolution method. Numbers in parentheses show the half-widths of $95 \%$ confidence intervals Bold numbers are the best estimates. Numbers in italics indicate a systematic difference from the corresponding reconvolved results. Bold italics indicate the best estimate but with a systematic difference.
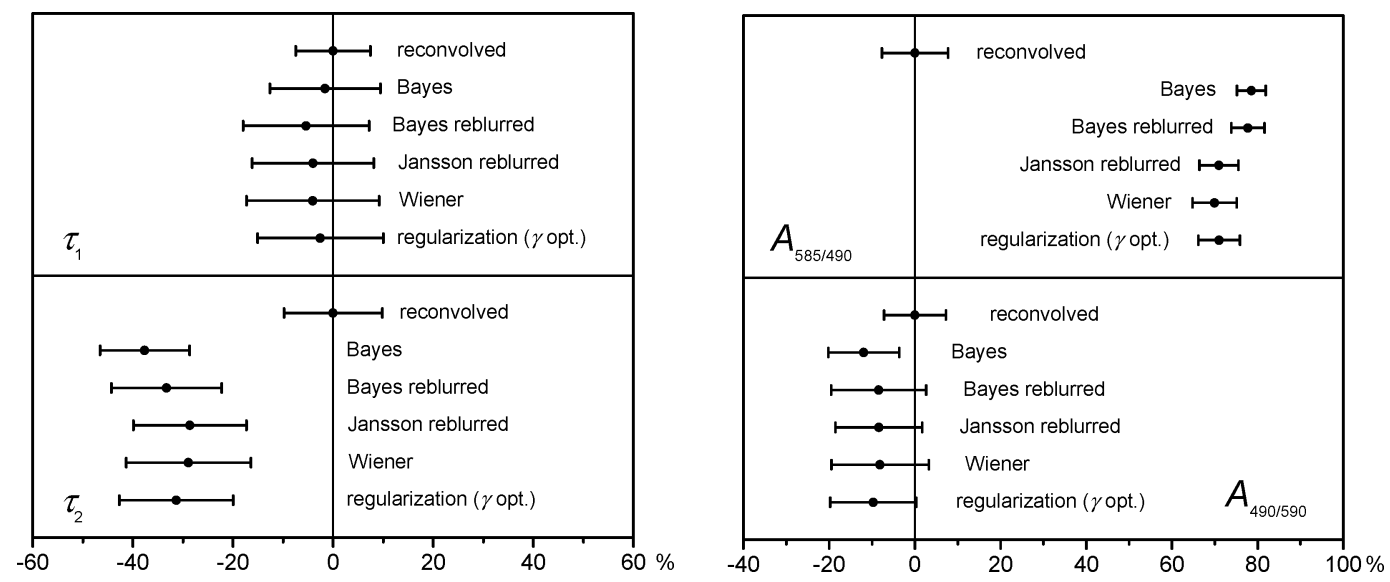

Figure 10. 95\% confidence intervals in percentage relative to the reconvolved parameter value for the two time constants $\tau_{1}$ and $\tau_{2}$ and two transient $\mathrm{Na}^{-*}$ absorbances $A_{585 / 490}$ and $A_{490 / 590}$ of the model function described in ref 40 from a global fit to the three deconvolved experimental curves for data sets obtained with different deconvolution methods.

methods, in a similar manner as the test results of the synthetic data in Figure 6. From this comparison and the data of Table 2 we can see that four of the seven parameters, $\tau_{1}, P_{490}, P_{615}$ and $A_{490 / 590}$, obtained from a fit of the model function to the deconvolved data sets are practically identical with those obtained from reconvolution (the corresponding confidence intervals overlap), but there is a systematic deviation in the case of the remaining three parameters $\tau_{2}, P_{585}$, and $A_{585 / 490}$. Whether it is a systematic error introduced by the nonparametric deconvolution, or a systematic distortion of the parameters obtained from reconvolution which is absent in those obtained from the deconvolved data, we cannot unquestionably decide. Anyway, on the basis of the evidence seen with simulated data, we have quite good confidence in believing that nonparametric deconvolution resulted in a smaller systematic distortion than in the case of parameter estimation from the reconvolution. It is also interesting to note that errors estimated from the deconvolved data are not that much different from those obtained from reconvolution; typically $20-50 \%$ higher only. Moreover, some spectrophysical parameters have a smaller uncertainty due to an efficient smoothing of the experimental error.

It should be added, however, that the parameters estimated from the three curves selected are certainly not as statistically accurate as the parameters inferred simultaneously from 22 curves in ref 40 , so this study does not really challenge the parameters reported in the original paper; rather it calls attention to the fact that, here again, nonparametric deconvolution might lead to a less distorted estimated parameter set.

A few curves detected between 1200 and $1400 \mathrm{~nm}$ show a small peaklike bump at very short time delays. This rather fast rise and immediate fast decay are not interpreted by the kinetic and photophysical model of ref 40 quantitatively; it is only supposed that this feature appears due to early time absorption and a subsequent fast decay to a weak excited-state absorption that shifts rapidly to the red via solvation. (See also Figure 3a,c of ref 41 , where the transient peak is more sharp due to the greater time resolution.) Using a nonparametric deconvolution method, we can nevertheless reconstruct an estimate of the original, undistorted kinetic curve to check the height of this initial feature. It turns out that a considerably large peak can be reconstructed using a simple Bayesian deconvolution of the reblurred data. As it can be seen from Figure 11, the peak is enhanced by a factor of more than 5 due to deconvolution only. This result shows that nonparametric deconvolution allows a much deeper insight into the nature of kinetics and/or photophysics of the femtochemical process even in a case when no detailed inference from the raw data can be obtained.

The case of 780/1250 nm data shown in Figure 11 reveals another interesting feature. The measured data set does not have a unimodal shape, but there are a very sharp and a rather flat peak involved. Accordingly, the Fourier transform of this curve should show a distinct high-amplitude region at some higher frequencies as well, in addition to the typical low-frequency behavior peaking at zero frequency. Obviously, the strategy of graphical optimization described above for a relatively flat unimodal function cannot be applied in this case. It should be modified so that after an initial decrease there should be an increase of the amplitude, and the usual monotonic decrease should be achieved following this increase. This feature, relatively rare among measured femtosecond kinetic traces, shows that one should be cautious to choose the applied strategy 

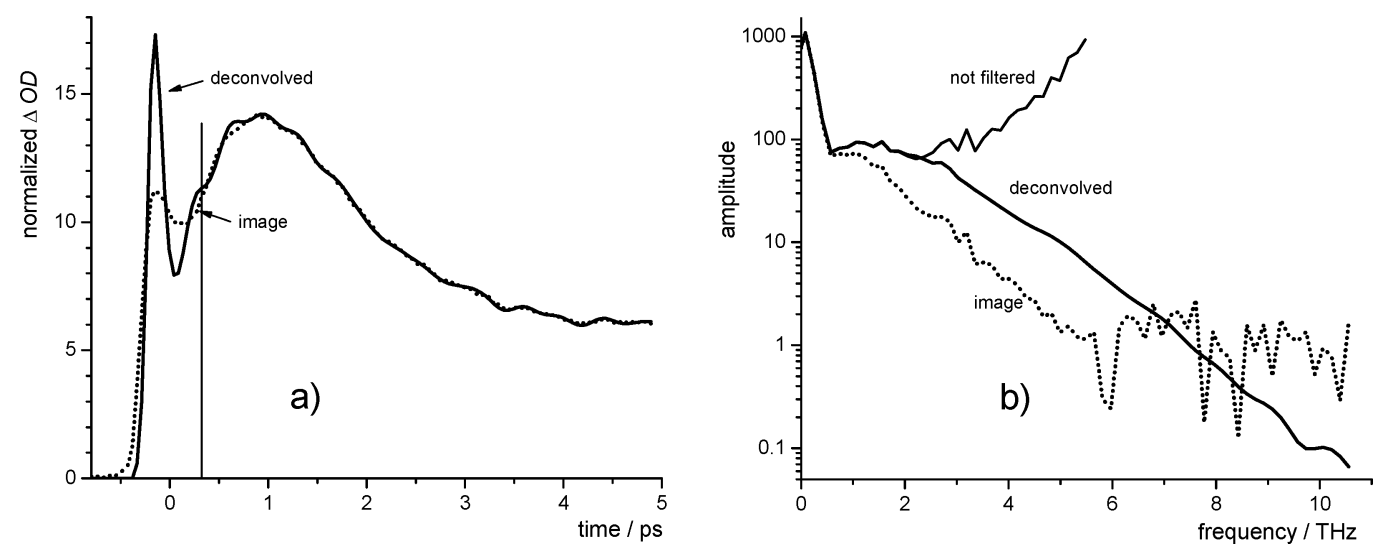

Figure 11. Dotted line: Experimental ultrafast kinetic curve at $780 \mathrm{~nm}$ excitation and $1250 \mathrm{~nm}$ detection wavelength (ref 40, Figure 3 , panel K). Solid line: Deconvolved curve obtained with a Bayesian iterative method of the reblurred data, using $350 \mathrm{fs}$ fwhm spread function. Panel a shows the time-domain data, while panel b shows the corresponding Fourier transforms in the frequency domain along with the solid line (marked "not filtered") showing the inverse filtered amplitude spectrum without additional noise filtering.

for a graphical optimization with actual measured data sets. A careful investigation of the Fourier transform of the measured function usually offers the guide for a proper optimization procedure. In this example, it is clear from the frequency spectrum marked "not filtered" in panel b that the onset of the undesirable noise amplification appears only after the slight increase in amplitude responsible for the initial sharp peak.

\section{Conclusion}

We have thoroughly examined the possibility of model-free deconvolution of femtosecond kinetic data using adaptations of iterative methods in the time domain and inverse filtering in the frequency domain with additional noise filters. We have found that the two major problems to solve are experimental noise and the steplike shape of many kinetic traces.

The steplike signal shape results in a fairly distorted Fourier transform, as the cyclic transformation introduces a sudden drop or rise in the data set, which leads to the appearance of virtual high-frequency components. The method of Gans and Nahman ${ }^{37}$ suggested in the signal processing literature avoids this problem by doubling the data set in a way that results in a periodic function without the sudden change. To treat the consequences of experimental noise which renders the solution of the convolution equation largely unstable, we have found powerful additional noise filters. In the time domain, the procedure called reblurring ${ }^{20,21}$ turned out to be a sufficient noise pretreatment to avoid the spurious behavior of the deconvolved data set during iterative deconvolution. This treatment consists of the convolution of both the measured signal and the effective pulse with the time-inverted pulse, and the subsequent deconvolution of the smoothed signal using the reblurred pulse, which is its autocorrelation. In the frequency domain, this pretreatment does not help, as inverse filtering is highly sensitive even to very small numerical noise. The solution in this case is a composite filter, i.e., the simultaneous application of a suitable noise filter with the inverse filter. Adaptive Wiener filtering ${ }^{31,32}$ and regularization $^{33,34,36}$ gave satisfactory results in efficiently reducing the noise while leading only to a very small distortion of the useful signal.

A thorough analysis of the numerical implementation of the above-mentioned methods with simulated synthetic data revealed the existence of optimal deconvolution to reconstruct the original undistorted data, and the good quality of the results as a basis of a statistical inference to get reliable parameters of the underlying physical models. It has also been found that model parameters obtained this way were less biased than those obtained with a classical reconvolution, i.e., inferring parameters from fitting measured data to the convolved model functions. However, we were unable to find any target functions that could be used to quantitatively indicate optimal filter parameters or iterative results in the case of experimental data when the true signal is not known. To treat this problem, we have developed a graphical method based on the observation of the frequencydomain behavior of the Fourier transforms of the deconvolution result, the measured data set and that of the simple inverse filtered result without any noise filtering. We have found that the visual observation of these curves provides rather robust deconvolution results. If a frequency sensitive deconvolution is needed, inverse filtering can be an appropriate choice. In the case if the efficiency of the deconvolution should be different depending on the amplitude, the time-domain iterative Jansson method with a suitably shaped relaxation function offers the best possibility.

We have also tested all the methods that could be used to deconvolve synthetic data sets to the deconvolution of real-life experimental data. ${ }^{40}$ It turned out that there can appear some new features of the measured data which were not appertaining to the synthetic data studied, but adaptation of the findings with the synthetic data are quite straightforward based on the actual shape of the experimental kinetic traces. In the case of an unresolved experimental data set, where the rather fast feature involved has no explanation yet, the model-free deconvolution has revealed a substantially different signal shape from that of the detected data.

We continue our efforts to find some more heuristic deconvolution methods, as for example the genetic algorithm that would enable the use of quantitative criteria to find an optimal deconvolved data set even if the original undistorted signal is unknown. It should also be mentioned that deconvolution methods implemented and analyzed can have a more wide application range than femtosecond chemistry. Whenever relatively slowly varying steplike functions are measured distorted by convolution, all the methods described here might be worth trying.

Acknowledgment. The authors thank Tamás Dabóczi for enlightening discussions concerning discrete Fourier transformations and inverse filtering. This work was supported by the National Research Fund of Hungary under Contract No. OTKA T 048725 . 
Supporting Information Available: A Visual Basic 6.0 program for inverse filtering of femtosecond kinetic data with a graphical interface, accompanied with a few examples of data (both simulated and experimental). This material is available free of charge via the Internet at http://pubs.acs.org.

\section{References and Notes}

(1) van Cittert, P. H. Z. Phys. 1931, 69, 298; Burger, H. C.; van Cittert, P. H. Z. Phys. 1933, 81, 428.

(2) Paley, R. C.; Wiener, N. Fourier Transforms in the Complex Domain; AMS Colloquium Publications: Providence, RI, 1934; Vol. 19

(3) Press, W. H.; Flannary, B. P.; Teukolsky, S. A.; Vetterling, W. T. Numerical Recipes - The Art of Scientific Computing; Cambridge University Press: Cambridge, 1986.

(4) Hamming, R. W. Digital Filters, 3rd ed.; Prentice Hall: Englewood Cliffs, NJ, 1989

(5) See, e.g.: Chase, W. J.; Hunt, J. W. J. Phys. Chem. 1975, 79, 2835.

(6) McKinnon, A. E.; Szabo, A. G.; Miller, D. R. J. Phys. Chem. 1977, $81,1564$.

(7) O'Connor, D. V.; Ware, W. R.; André, J. C. J. Phys. Chem. 1979, 83, 1333.

(8) See, e.g.: Bernstein, R. B.; Zewail, A. H. Chem. Eng. News 1988 (Nov. 7), 24-43. Zewail, A. H. Science 1988, 242, 1645. Zewail, A. H. J. Chem. Soc., Faraday Trans. 2 1989, 85, 1221. Simon, J. D., Ed. Ultrafast Dynamics of Chemical Systems; Kluwer Academic Publishers: Dordrecht, 1994. Zewail, A. H. J. Phys. Chem. 2000, 104, 5660.

(9) Bányász, A.; Mátyus, E.; Keszei, E. Radiat. Phys. Chem. 2005, 72,235 .

(10) Bányász, A.; Dancs, G.; Keszei, E. Radiat. Phys. Chem. 2005, 74, 139.

(11) Keszei, E.; Murphrey, T. H.; Rossky, P. J. J. Phys. Chem. 1995, $99,22$.

(12) For a mathemathical description, see, e.g.: Zayed, A. I. Handbook of Function and Generalized Function Transformations; CRC Press: Boca Raton, 1996; p 233. Relevant implications are described in the following: Donoho, D. L.; Stark, P. B. SIAM J. Appl. Math. 1989, 49, 906.

(13) Henriksen, N. E.; Engel, V. J. Chem. Phys. 1999, 111, 10469

(14) See, e.g., p 383 of ref 3.

(15) It is appropriate to note here that the width of the effective pulse is at least $\sqrt{2}$ times that of the pump pulse, as the probe pulse is usually generated from the same initial laser pulse without compression. The group velocity mismatch further increases the temporal width of the effective pulse. To our experience, the effective pulse width is usually close to twice the pump pulse width.
(16) Habenicht, A.; Hjelm, J.; Mukhtar, E.; Bergström, F.; Johansson, L. B.-A. Chem. Phys. Lett. 2002, 354, 367.

(17) Jansson, P. A., Ed. Deconvolution of Images and Spectra, 2nd ed.; Academic Press: San Diego, 1997.

(18) Schafer, R. W.; Merserau, R. M.; Richards, M. A. Proc. IEEE 1981, 69,432

(19) Biemond, J.; Lagendijk, R. L.; Mersereau, R. M. Proc. IEEE 1990 78,856

(20) Kawata, S.; Ichioka, Y. J. Opt. Soc. Am. 1980, 70, 762.

(21) Kawata, S.; Ichioka, Y. J. Opt. Soc. Am. 1980, 70, 768.

(22) Amini, A. M. Appl. Opt. 1995, 34, 1878.

(23) Jansson, P. A.; Hunt, R. H.; Pyler, E. K. J. Opt. Soc. Am. 1970, 60,692 .

(24) Gold, R. An Iterative Unfolding Method for Response Matrices; AEC Research and Development Report ANL-6984; Argonne National Laboratory: Argonne, IL, 1964.

(25) Xu, C.; Aissaoui, L.; Jacquey, S. J. Opt. Soc. Am. A 1994, 11, 2804

(26) Richardson, W. H. J. Opt. Soc. Am. 1972, 62, 55.

(27) Kennett, T. J.; Prestwich, W. V.; Robertson, A. Nucl. Instrum. Methods 1978, 151, 285, 293. Prozesky, V. M.; Padayachee, J.; Fischer, R.; Von der Linden, W.; Dose, V.; Ryan, C. G. Nucl. Instrum. Methods Phys. Res. B 1979, 130, 113

(28) Crilly, P. B. IEEE Trans. Instrum. Meas. 1991, 40, 558.

(29) Pananakis, D.; Abel, E. W. Thermochim. Acta 1998, 315, 107.

(30) André, J. C.; Vincent, L. M.; O'Connor, D.; Ware, W. R. J. Phys. Chem. 1979, 83, 2285.

(31) Gobbel, G. T.; Fike, J. R. Phys. Med. Biol. 1994, 39, 1833

(32) Bates, J. H. T. IEEE Trans. Biomed. Eng. 1991, 38, 1262.

(33) Tikhonov, A. N.; Arsenin, V. Y. Solutions of Ill-posed Problems;

V. H. Winston \& Sons: Washington, DC, 1977.

(34) Dabóczi, T.; Kollár, I. IEEE Trans. Instrum. Meas. 1996, 45, 417.

(35) Madden, F. N.; Godfrey, K. R.; Chappell, M. J.; Hovorka, R.; Bates, A. R. J. Pharmacokinet. Biopharm. 1996, 24, 283. 281.

(37) Gans, W. L.; Nahman, N. S. IEEE Trans. Instrum. Meas. 1982, $31,97$.

(38) Waldmeyer, J. IEEE Trans. Instrum. Meas. 1980, 29, 36.

(39) Marquardt, D. W. J. Soc. Ind. Appl. Math. 1963, 11, 431; see also ref 3 of the present paper.

(40) Barthel, E. R.; Martini, I. B.; Keszei, E.; Schwartz, B. J. J. Chem. Phys. 2003, 118, 5916.

(41) Wang, Z.; Shoshana, O.; Hou, B.; Ruhman, S. J. Phys. Chem. A 2003, 107, 3009 . 